\title{
POLY-SYMPLECTIC GROUPOIDS AND POLY-POISSON STRUCTURES
}

\author{
NICOLAS MARTINEZ
}

\begin{abstract}
We introduce poly-symplectic groupoids, which are natural extensions of symplectic groupoids to the context of poly-symplectic geometry, and define poly-Poisson structures as their infinitesimal counterparts. We present equivalent descriptions of poly-Poisson structures, including one related with AVDirac structures. We also discuss symmetries and reduction in the setting of poly-symplectic groupoids and poly-Poisson structures, and use our viewpoint to revisit results and develop new aspects of the theory initiated in [19].
\end{abstract}

\section{CONTEnts}

1. Introduction

2. Poly-symplectic groupoids

2.1. Poly-symplectic structures

2.2. Multiplicative forms and poly-symplectic groupoids

2.3. Infinitesimal data of poly-symplectic groupoids

3. Poly-Poisson structures

3.1. Definition

3.2. Poly-Poisson structures and poly-symplectic groupoids

3.3. Poly-symplectic foliation

3.4. Relation with AV-Dirac structures 13

4. Symmetries and reduction $\quad 15$

4.1. Poly-Poisson actions 15

4.2. Hamiltonian actions on poly-symplectic manifolds 17

4.3. Reduction and integration 20

References 23

\section{INTRODUCTION}

Poly-symplectic structures arise in the geometric formulation of Classical Field Theories in the same way that symplectic structures appear in the Hamiltonian formalism of classical mechanics [18. More precisely, poly-symplectic structures are $\mathbb{R}^{k}$-valued 2-form, which are closed and satisfy a nondegeneracy condition, in such a way that they concide with usual symplectic forms when $k=1$. Poly-symplectic geometry has been studied in recent years by several authors, including [2, 3, 21, 23, 31]; see also [16, 20, 22, 29, 33] for further connections with physics.

In recent work [19, D. Iglesias J.C. Marrero and M. Vaquero introduced a generalization of Poisson structure by considering the inverse structures of poly-symplectic 
forms, analogous to the way Poisson structures are defined from symplectic forms. In this paper, we give a new viewpoint and study new aspects of the work in [19] by considering a slight variation of their definition of poly-Poisson structure. Our definiton relies on the relationship between symplectic groupoids and Poisson manifolds [35, 11, but now in the setting of poly-symplectic groupoids, which are natural extensions of symplectic groupoids to poly-symplectic geometry.

Similarly to symplectic groupoids, poly-symplectic groupoids are defined by a polysymplectic form on a Lie groupoid satisfying a compatibility condition, which says that the poly-symplectic form is multiplicative (in the sense of (2.2) below). One of the main properties of symplectic groupoids is that they are the global versions of Poisson structures (see [35, 11]), that is, the manifold of objects of a symplectic groupoid is endowed with a Poisson structure whose corresponding Lie algebroid is isomorphic to the Lie algebroid of the groupoid. Moreover, the Poisson structure is uniquely determined by the condition that the target map is a Poisson morphism. Starting with a poly-symplectic groupoid, the corresponding infinitesimal geometric structure is what we identify and call poly-Poisson structure. In other words, the poly-Poisson structures we introduce here relate to poly-symplectic groupoids exactly in the same way that Poisson structures relate to symplectic groupoids. A similar idea in the context of multi-symplectic geometry (see [9, 10]) is studied in [8].

The notion of $k$-poly-Poisson structure arising in this way is slightly less general than the one given in [19], but contains the essential examples of the theory. Moreover, for $k=1$, the notion agrees with ordinary Poisson structures (in contrast with the more general definition of [19]). From our viewpoint to poly-Poisson structures, we will revisit some results in [19] and extend known facts about Poisson structures, e.g., concerning their underlying Lie algebroids and foliations. Also, following the description of Poisson structures as particular cases of Dirac structures [12, we discuss an analogous picture for poly-Poisson structures. In this case, however, Dirac structures are not enough, and we must consider AV-Courant algebroids and a suitable extension of AV-Dirac structures, as in [24].

Poly-symplectic manifolds $M$ equipped with symmetries given by a Lie group $\mathbb{G}$ induce, under suitable regularity conditions, a quotient poly-Poisson structure on the manifold $M / \mathbb{G}$. In order to find poly-symplectic groupoids integrating such quotients, we need to discuss some aspects of hamiltonian actions and MarsdenWeinstein reduction in poly-symplectic geometry, see e.g. [18, 27]. This allows us to extend some constructions in [30, 17] and [7, and show that the symmetries $\mathbb{G}$ of an integrable poly-Poisson manifold can be lifted to hamiltonian symmetries of its integrating (source-simply-connected) poly-symplectic groupoid, and that its poly-symplectic reduction at level zero is a poly-symplectic groupoid integrating the quotient poly-Poisson structure on $M / \mathbb{G}$.

There are several aspects of the approach to higher Poisson structures considered in this paper that we plan to pursue in future work, including the study of normal forms (see [2, 28] and the more recent work in [16]), the geometry of the corresponding higher versions of Dirac structures, and the potential connections with Field Theory. 
This paper is organized as follows: In Section 2 we introduce poly-symplectic groupoids. The key result of this section, which generalizes [8, Prop. 4.1], is Proposition 2.4, where we obtain the relation between global and infinitesimal objects. Poly-Poisson structures are defined in Section 3, where we discuss their Lie algebroid structure, the underlying foliation, together with their relation with poly-symplectic groupoids via integration. Poly-Poisson structures are illustrated with some examples from [19]. At the end we give a different way to describe poly-Poisson structures related to AV-Dirac structures [24]. Section 4 is devoted to the study of symmetries of poly-Poisson structures and hamiltonian actions on poly-symplectic manifolds, see Theorem 4.1 and Prop. 4.4. Finally, applying the hamiltonian reduction, we describe integrations of quotients of an integrable poly-Poisson manifold.

Acknowledgments: I would like to thanks Henrique Bursztyn for enlightening and helpful discussions and Juan Carlos Marrero and David Iglesias for their comments on this notes. This work was supported by a fellowship from CNPq.

Notation: Lie algebroids will be denoted by $A \rightarrow M$, with anchor map $\rho: A \rightarrow T M$ and bracket $[\cdot, \cdot]$. For a Lie groupoid $\mathcal{G}$ over $M$, the source and target maps will be $s, t: \mathcal{G} \rightarrow M, \epsilon: M \rightarrow \mathcal{G}$ denotes the unit map, inv $: \mathcal{G} \rightarrow \mathcal{G}$ is the inversion map, and the groupoid multiplication is $m: \mathcal{G}_{(2)} \rightarrow \mathcal{G}$, where the space of composable arrows is $\mathcal{G}_{(2)}:=\mathcal{G} \times{ }_{s, t} \mathcal{G}=\{(g, h) \in \mathcal{G} \times \mathcal{G} \mid t(h)=s(g)\}$. The right and left translation on the groupoid are $R_{g}, L_{g}$, respectively, for $g \in \mathcal{G}$.

For a vector space $V$, we will denote by $\oplus_{(k)} V$ the $k$-fold direct sum of $V$, or equivalently, the space $V \otimes \mathbb{R}^{k}$. On vector spaces we will use two different annihilator spaces. For a vector subspaces $W$ of a vector space $V$, we will denote by $\operatorname{Ann}(W)$ the space of elements on $V^{*}$ vanishing on $W$. For any subspace $S$ of $\oplus_{(k)} V^{*}, S^{o}$ stands for the space of elements on $V$ which annihilate the elements of $S$, i.e $S^{\circ}=$ $\{v \in V \mid \alpha(v)=0$ for all $\alpha \in S\}$. This notation will be used, more generally, for vector bundles $E \rightarrow M$ rather than vector spaces.

The coadjoint action $\mathrm{Ad}^{*}: \mathbb{G} \rightarrow \operatorname{End}\left(\mathfrak{g}^{*}\right)$ of a Lie group $\mathbb{G}$ on the dual of its Lie algebra $\mathfrak{g}$ induces a diagonal coadjoint action of $\mathbb{G}$ on the product $\mathfrak{g}_{(k)}^{*}$, and we keep the notation $\mathrm{Ad}^{*}$ to this action, i.e., $\operatorname{Ad}_{g}^{*}\left(\zeta_{1}, \ldots, \zeta_{k}\right)=\left(\operatorname{Ad}_{g}^{*} \zeta_{1}, \ldots, \operatorname{Ad}_{g}^{*} \zeta_{k}\right)$.

\section{Poly-SympleCtic GROUPOIDS}

In this section we will recall the concept of poly-symplectic manifold (see e.g. [18, 19, 2]) and introduce poly-symplectic groupoids, which will guide us towards poly-Poisson structures.

2.1. Poly-symplectic structures. A $k$-poly-symplectic form on a manifold $M$ is a an $\mathbb{R}^{k}$-valued differential form $\omega \in \Omega^{2}\left(M, \mathbb{R}^{k}\right)$ which is closed and nondegenerate, in the sense that the induced bundle map

$$
\omega^{b}: T M \rightarrow T^{*} M \otimes \mathbb{R}^{k}
$$

is injective $(\operatorname{ker}(\omega)=\{0\})$. Writing $\omega$ in terms of its components, $\omega=\left(\omega_{1}, \ldots, \omega_{k}\right)$, it is poly-symplectic if and only if each $\omega_{j} \in \Omega^{2}(M)$ is closed and

$$
\cap_{j=1}^{k} \operatorname{ker}\left(\omega_{j}\right)=\{0\} \text {. }
$$


One way to obtain examples of poly-symplectic structures is the following. Let $M$ be a manifold endowed with $k$ surjective, submersion maps $p_{j}: M \rightarrow M_{j}$, such that $\cap_{j=1}^{k} \operatorname{ker}\left(d p_{j}\right)=\{0\}$. If each $M_{j}$ is equipped with a $l_{j}$-poly-symplectic form $\omega_{j}$, then

$$
\omega=\left(p_{1}^{*} \omega_{1}, \ldots, p_{k}^{*} \omega_{k}\right)
$$

is an $l$-poly-symplectic form on $M$, where $l=l_{1}+\ldots+l_{k}$. In particular, if $\left(M_{j}, \omega_{j}\right)$ is an $l_{j}$-poly-symplectic manifold, $j=1, \ldots, k$, this construction endows $M:=M_{1} \times$ $\cdots \times M_{k}$ with an $l$-poly-symplectic structure, for $l=l_{1}+\ldots+l_{k}$. This shows that the product of $k$ symplectic manifolds naturally carries a $k$-poly-symplectic structure.

The following is a particular case of interest in classical field theory [18]:

Example 2.1. ( $k$-covelocities on a manifold) Recall that any cotangent bundle $T^{*} Q$ has a canonical symplectic form $\omega_{\text {can }}$. The manifold of $k$-covelocities is the Whitney sum

$$
\oplus_{(k)} T^{*} Q=T^{*} Q \oplus \stackrel{(k}{*} \oplus T^{*} Q,
$$

which is equipped with the natural projections $\operatorname{pr}_{j}: \oplus_{(k)} T^{*} Q \rightarrow T^{*} Q$. It is clear that $\cap_{j=1}^{k} \operatorname{ker}\left(d \mathrm{pr}_{j}\right)=\{0\}$, and

$$
\omega:=\left(\operatorname{pr}_{1}^{*} \omega_{c a n}, \ldots, \operatorname{pr}_{k}^{*} \omega_{c a n}\right) \in \Omega^{2}\left(\oplus_{(k)} T^{*} Q, \mathbb{R}^{k}\right)
$$

is a $k$-poly-symplectic form.

Other examples of poly-symplectic structures are discussed e.g. in [18, 19, 32].

2.2. Multiplicative forms and poly-symplectic groupoids. We now consider poly-symplectic structures on Lie groupoids. Let $\mathcal{G}$ be a Lie groupoid over $M$.

A differential form $\theta \in \Omega^{r}(\mathcal{G})$ is called multiplicative if it satisfies

$$
m^{*} \theta=\operatorname{pr}_{1}^{*} \theta+\operatorname{pr}_{2}^{*} \theta,
$$

where $\operatorname{pr}_{i}: \mathcal{G} \times_{s, t} \mathcal{G} \rightarrow \mathcal{G}$ are the projection maps. Note that condition (2.2) still makes sense for $\mathbb{R}^{k}$-valued forms $\theta=\left(\theta_{1}, \ldots, \theta_{k}\right)$, and it simply says that each component $\theta_{i}$ is multiplicative.

Recall that a symplectic groupoid is a Lie groupoid $\mathcal{G} \rightrightarrows M$ endowed with a multiplicative symplectic form $\omega \in \Omega^{2}(\mathcal{G})$, see e.g. [11, 35]. A direct generalization leads to

Definition 2.1. A k-poly-symplectic groupoid is a Lie groupoid $\mathcal{G} \rightrightarrows M$ together with a $k$-poly-symplectic form $\omega=\left(\omega_{1}, \ldots, \omega_{k}\right) \in \Omega^{2}\left(\mathcal{G}, \mathbb{R}^{k}\right)$ satisfying (2.2). More explicitly, each $\omega_{j} \in \Omega^{2}(\mathcal{G})$ is closed, multiplicative, and $\cap_{j=1}^{k} \operatorname{ker}\left(\omega_{j}\right)=\{0\}$.

Suppose that $\mathcal{G}_{j} \rightrightarrows M_{j}$ are $l_{j}$-poly-symplectic groupoids, $j=1, \ldots, k$. As discussed in Section [2.1, we can verify that if a Lie groupoid $\mathcal{G}$ is equipped with surjective submersions $p_{j}: \mathcal{G} \rightarrow \mathcal{G}_{j}, j=1, \ldots, k$, which are groupoid morphisms and satisfy $\cap_{j} \operatorname{ker}\left(d p_{j}\right)=\{0\}$, then $\omega=\left(p_{1}^{*} \omega_{1}, \ldots, p_{k}^{*} \omega\right) \in \Omega^{2}\left(\mathcal{G}, \mathbb{R}^{k}\right)$ makes $\mathcal{G}$ into an $l$-poly-symplectic groupoid, for $l=l_{1}+\ldots+l_{k}$. Here we use the fact that the pullback of a multiplicative form by a groupoid morphism is again multiplicative. In particular, we have:

Proposition 2.2. The direct product of symplectic groupoids $\left(\mathcal{G}_{j}, \omega_{j}\right), j=1, \ldots, k$, naturally carries a multiplicative $k$-poly-symplectic structure given by

$$
\omega=\left(\operatorname{pr}_{1}^{*} \omega_{1}, \ldots, \operatorname{pr}_{k}^{*} \omega_{k}\right)
$$


where $\operatorname{pr}_{j}: \mathcal{G}_{1} \times \ldots \times \mathcal{G}_{k} \rightarrow \mathcal{G}_{j}$ is the natural projection.

More conceptually, multiplicative poly-symplectic forms are very special cases of multiplicative forms with values in representations, as in [15]. Given a Lie groupoid $\mathcal{G} \rightrightarrows M$ and a vector bundle $E \rightarrow M$, consider the pullback bundle $t^{*} E \rightarrow \mathcal{G}$. An $E$-valued $r$-form on $\mathcal{G}$ is an element $\theta \in \Omega^{r}\left(\mathcal{G}, t^{*} E\right)$. If $E$ is a representation of $\mathcal{G}$ (see [25]), we say that $\theta \in \Omega^{r}\left(\mathcal{G}, t^{*} E\right)$ is multiplicative if for all composable arrows $(g, h) \in \mathcal{G} \times_{s, t} \mathcal{G}$ we have

$$
\left(m^{*} \theta\right)_{(g, h)}=\operatorname{pr}_{1}^{*} \theta+g \cdot\left(\operatorname{pr}_{2}^{*} \theta\right)
$$

where $m, \operatorname{pr}_{1}, \mathrm{pr}_{2}$ are as in (2.2). It is clear that for the trivial bundle $E=\mathbb{R}^{k} \times M$, equipped with the trivial representation, this recovers the notion of multiplicative $\mathbb{R}^{k}$-valued forms previously discussed.

For later use, we observe the $E$-valued version of the equations in [4, Lemma 3.1(i)]:

Lemma 2.3. If $\theta \in \Omega^{k}\left(\mathcal{G}, t^{*} E\right)$ is multiplicative then

$$
\epsilon^{*} \theta=0, \text { and } \theta_{g}=-g \cdot\left(\operatorname{inv}^{*} \theta_{\operatorname{inv}(g)}\right)
$$

for all $g \in \mathcal{G}$.

Proof. Define the map $(I d \times \operatorname{inv})(g):=\left(g, g^{-1}\right)$ from $\mathcal{G}$ to $\mathcal{G}_{(2)}$. If we apply the pull-back of $(I d \times \mathrm{inv})$ to Equation (2.3) and recall that $\epsilon \circ t=m \circ(I d \times$ inv $)$, we obtain:

$$
\begin{aligned}
t^{*} \epsilon^{*} \theta_{\epsilon(t(g))} & =(I d \times \operatorname{inv})^{*}\left(m^{*} \theta\right)_{\left(g, g^{-1}\right)}=\theta_{g}+(I d \times \operatorname{inv})^{*}\left(g \cdot\left(p r_{2}^{*} \theta_{g^{-1}}\right)\right) \\
& =\theta_{g}+g \cdot\left((I d \times \operatorname{inv})^{*} p r_{2}^{*} \theta_{g^{-1}}\right) .
\end{aligned}
$$

Therefore

$$
t^{*} \epsilon^{*} \theta_{\epsilon(t(g))}=\theta_{g}+g \cdot\left(\text { inv }^{*} \theta_{g^{-1}}\right) .
$$

If in particular we fix $g=\epsilon(m)$ for some $m \in M$ and take the pull-back by the unit map in (2.5), we conclude that $\epsilon^{*} \theta=0$. Using this identity and (2.5), it follows that $\theta_{g}+g \cdot\left(\operatorname{inv}^{*} \theta_{g^{-1}}\right)=0$.

2.3. Infinitesimal data of poly-symplectic groupoids. It is well known that Poisson structures are the infinitesimal counterparts of symplectic groupoids, see e.g. [11, 35]. We will now discuss the infinitesimal counterpart of poly-symplectic groupoids, in the spirit of [8, which leads to a generalization of Poisson structures in poly-symplectic geometry.

Let $A \rightarrow M$ denote the Lie algebroid of a Lie groupoid $\mathcal{G} \rightrightarrows M$, with anchor $\rho$ : $A \rightarrow T M$ and bracket $[\cdot, \cdot]$ on $\Gamma(A)$. Recall from [1, 6, 4] that a closed multiplicative $r$-form $\theta$ on $\mathcal{G}$ is infinitesimally described by a bundle map (over the identity)

$$
\mu: A \rightarrow \wedge^{r-1} T^{*} M
$$

satisfying the conditions

$$
\begin{aligned}
i_{\rho(u)} \mu(v) & =-i_{\rho(v)} \mu(u), \quad \forall u, v \in A \\
\mu([u, v]) & =\mathcal{L}_{\rho(u)} \mu(v)-i_{\rho(v)} d \mu(u), \quad \forall u, v \in \Gamma(A) .
\end{aligned}
$$

The map $\mu$ is related to $\theta$ via

$$
i_{u^{R}} \theta=t^{*}(\mu(u)),
$$


for $u \in \Gamma(A)$, where $u^{R}$ denotes the right-invariant vector field on $\mathcal{G}$ defined by $u$. For source-simply-connected Lie groupoids, $\mu$ and $\theta$ completely determine one another.

It follows that a closed multiplicative $\mathbb{R}^{k}$-valued 2 -form $\omega=\left(\omega_{1}, \ldots, \omega_{k}\right) \in \Omega^{2}\left(\mathcal{G}, \mathbb{R}^{k}\right)$ infinitesimally corresponds to a bundle map

$$
\mu=\left(\mu_{1}, \ldots, \mu_{k}\right): A \rightarrow \oplus_{(k)} T^{*} M
$$

satisfying the same equations (2.6) an (2.7), which simply means the equations are satisfied componentwise, i.e., each $\mu_{j}: A \rightarrow T^{*} M$ is a closed IM 2-form. For the complete infinitesimal description of a multiplicative poly-symplectic form, it remains to express the non-degeneracy condition $\cap_{j=1}^{k} \operatorname{ker}\left(\omega_{j}\right)=\{0\}$ in terms of the map $\mu$ in (2.9). We will do that in the more general framework of multiplicative forms on $\mathcal{G}$ with values in representations $E \rightarrow M$.

The infinitesimal version of multiplicative $E$-valued $r$-forms on a Lie groupoid $\mathcal{G}$ was studied in [15], where it is proven that (under the usual source-simplyconnectedness condition on $\mathcal{G}$ ) such forms $\theta$ are in 1-1 correspondence with pairs of maps $(D, \mu)$,

$$
D: \Gamma(A) \rightarrow \Omega^{r}(M, E), \quad \mu: A \rightarrow \wedge^{r-1} T^{*} M \otimes E,
$$

satisfying suitable conditions (that we will not need explicitly), see [15, Sec. 2.2]. We will only need the following facts about the infinitesimal data $(D, \mu)$. First, the relation between the bundle map $\mu$ and the multiplicative $E$-valued form $\theta$ is a direct generalization of that in (2.8): indeed, using [4, Eqs. (3.1)-(3.3)], it follows that the second equation of [15, (2.4)] is equivalent to

$$
i_{u^{R}} \theta=t^{*}(\mu(u)) .
$$

Second, when $E=\mathbb{R}^{k}$ is the trivial representation and the multiplicative form $\theta$ is closed, then $D$ is determined by $\mu$, in fact $D=d \mu$ (see [6]); so in this case one only needs $\mu$ for the infinitesimal description of $\theta$.

We say that an $r$-form $\theta \in \Omega^{r}\left(\mathcal{G}, t^{*} E\right)$ is non-degenerate when the map

$$
\theta^{b}: T \mathcal{G} \rightarrow \wedge^{r-1} T^{*} \mathcal{G} \otimes t^{*} E, \quad X \mapsto i_{X} \theta
$$

has trivial kernel. When $\theta$ is multiplicative, we have the following infinitesimal description of this property.

Proposition 2.4. Consider $\theta \in \Omega^{r}\left(\mathcal{G}, t^{*} E\right)$ a multiplicative E-valued $r$-form on a Lie groupoid $\mathcal{G}$, and let $\mu: A \rightarrow \wedge^{r-1} T^{*} M \otimes E$ be such that (2.10) holds. Then $\theta$ is nondegenerate if and only if

$$
\operatorname{ker}(\mu)=\{0\}, \quad \text { and }(\operatorname{Im}(\mu))^{\circ}=\{0\},
$$

where $(\operatorname{Im}(\mu))^{\circ}=\left\{X \in T M \mid i_{X} \mu(u)=0\right.$ for all $\left.u \in A\right\}$.

Proof. The proof uses the relation (2.10) and follows the same idea of [8, Prop. 4.1]. We recall the details for the reader's convenience.

First we suppose that conditions (2.11) hold for $\mu$ and take $X \in T_{g} \mathcal{G}$ in the kernel of the multiplicative form. We get that $d t X=0$ because $i_{X} t^{*}(\mu(u))=0$ for all $u \in A$ (from (2.10) $)$, hence $X$ is tangent to the $t$-fibers, which implies the existence of $v \in A$ for which $X=v_{g}^{L}=d_{g} \operatorname{inv}\left(v_{g}^{R}\right)$. As consequence of the second equation in (2.4) and (2.10), we see that $-g \cdot\left(s^{*}(\mu(v))\right)=i_{v_{g}^{L}} \theta_{g}=i_{X} \theta_{g}=0$ for any $g \in \mathcal{G}$, hence $s^{*}(\mu(v))=0$. This shows that $v \in \operatorname{ker}(\mu)$, therefore $X=v_{g}^{L}=0$. 
For the other direction, let $u \in \operatorname{ker}(\mu)$. Then $i_{u^{R}} \theta=t^{*}(\mu(u))=0$, which implies $u^{R}=0$ by nondegeneracy of the form, thus the first condition in (2.11) holds. Now fixing $X \in(\operatorname{Im}(\mu))_{m}^{\circ}$ for $m \in M$, (2.10) implies that $i_{u} i_{X} \theta=0$ for all $u \in A_{m}$. The splitting $T_{m} \mathcal{G}=T_{m} M \oplus A_{m}$ allows us to write $Z_{j}=X_{j}+u_{j} \in T_{m} \mathcal{G}, j=1, \ldots, r-1$, and the multilinearity of $\theta$ implies that

$$
i_{Z_{r-1}} \ldots i_{Z_{1}} i_{X} \theta=i_{X_{r-1}} \ldots i_{X_{1}} i_{X} \theta
$$

because the other terms vanish from the fact that $i_{u} i_{X} \theta=0$ for all $u \in A_{m}$. Now the first condition in (2.3) implies that $i_{Z_{r-1}} \ldots i_{Z_{1}} i_{X} \theta=0$ for all $Z_{j} \in T_{m} \mathcal{G}$, hence $X=0$.

For the trivial representation $E=M \times \mathbb{R}$ and forms of arbitrary degree $r$, Proposition 2.4 recovers [8, Prop. 4.1]. For the trivial representation $E=M \times \mathbb{R}^{k}$ and $r=2$, we obtain the infinitesimal description of multiplicative $k$-poly-symplectic forms.

Corollary 2.5. Let $\mathcal{G} \rightrightarrows M$ be a source-simply-connected groupoid. Then there is a one-to-one correspondence between multiplicative poly-symplectic forms $\omega \in$ $\Omega^{2}\left(\mathcal{G}, \mathbb{R}^{k}\right)$ and bundle maps $\mu: A \rightarrow \oplus_{(k)} T^{*} M$ satisfying (2.6), (2.7) and (2.11) via the relation $i_{u^{R}} \omega=t^{*}(\mu(u))$, for all $u \in \Gamma(A)$.

Given a Lie algebroid $A \rightarrow M$, we see from Corollary 2.5 that bundle maps $\mu: A \rightarrow \oplus_{(k)} T^{*} M$ satisfying (2.6), (2.7) and (2.11) are the infinitesimal counterparts of multiplicative poly-symplectic forms on Lie groupoids. So we refer to these objects as IM poly-symplectic forms, where "IM" stands for "infinitesimally multiplicative". We say that two IM poly-symplectic forms $\mu: A \rightarrow \oplus_{(k)} T^{*} M$ and $\mu^{\prime}: A^{\prime} \rightarrow$ $\oplus_{(k)} T^{*} M$ are equivalent if there is a Lie algebroid isomorphism $\varphi: A \rightarrow A^{\prime}$ such that $\mu=\mu^{\prime} \circ \varphi$. Under the equivalence in Corollary 2.5, they correspond to isomorphic poly-symplectic groupoids.

We will now use the infinitesimal geometry of poly-symplectic groupoids described in Corollary [2.5 to provide a new viewpoint to [19].

\section{Poly-Poisson structures}

3.1. Definition. The notion of poly-Poisson structure that we now introduce is a slight modification of that in [19].

Definition 3.1. A $k$-poly-Poisson structure on a manifold $M$ is a pair $(S, P)$, where $S \rightarrow M$ is a vector subbundle of $\oplus_{(k)} T^{*} M$ and $P: S \rightarrow T M$ is a vector-bundle morphism (over the identity) such that

(i) $i_{P(\bar{\eta})} \bar{\eta}=0$, for all $\bar{\eta} \in S$,

(ii) $S^{\circ}=\left\{X \in T M \mid i_{X} \bar{\eta}=0, \forall \bar{\eta} \in S\right\}=\{0\}$,

(iii) the space of section $\Gamma(S)$ is closed under the bracket

$\lfloor\bar{\eta}, \bar{\gamma}\rfloor:=\mathcal{L}_{P(\bar{\eta})} \bar{\gamma}-\mathcal{L}_{P(\bar{\gamma})} \bar{\eta}+d\left(i_{P(\bar{\gamma})} \bar{\eta}\right)=\mathcal{L}_{P(\bar{\eta})} \bar{\gamma}-i_{P(\bar{\gamma})} d \bar{\eta}, \quad$ for $\bar{\gamma}, \bar{\eta} \in \Gamma(S)$,

and the restriction of this bracket to $\Gamma(S)$ satisfies the Jacobi identity.

We will call the triple $(M, S, P)$ a $k$-poly-Poisson manifold.

We observe that the bracket (3.1) is skew-symmetric (by condition (i)) and satisfies the Leibniz rule:

$$
\lfloor\bar{\eta}, f \bar{\gamma}\rfloor=f\lfloor\bar{\eta}, \bar{\gamma}\rfloor+\left(\mathcal{L}_{P(\bar{\eta})} f\right) \bar{\gamma}
$$


for all $\bar{\eta}, \bar{\gamma} \in \Gamma(S)$ and $f \in C^{\infty}(M)$. It follows that, for a poly-Poisson manifold $(M, S, P)$, the vector bundle $S \rightarrow M$ is a Lie algebroid with bracket (3.1) and anchor map $P: S \rightarrow T M$. Since for any Lie algebroid the anchor map preserves Lie brackets, we have that

$$
P(\lfloor\bar{\eta}, \bar{\gamma}\rfloor)=[P(\bar{\eta}), P(\bar{\gamma})], \quad \forall \bar{\eta}, \bar{\gamma} \in \Gamma(S) .
$$

Remark 3.2. In (iii) of Def. [3.1, assuming that $\Gamma(S)$ is closed under the bracket (3.1), we can replace the condition on the Jacobi identity by the bracket-preserving property (3.2). Indeed, if (3.2) holds and for $\bar{\eta}, \bar{\lambda}, \bar{\gamma} \in \Gamma(S)$, then

$$
\begin{aligned}
\lfloor\lfloor\bar{\eta}, \bar{\gamma}\rfloor, \bar{\lambda}\rfloor & +\lfloor\bar{\gamma},\lfloor\bar{\eta}, \bar{\lambda}\rfloor\rfloor=\mathcal{L}_{[P(\bar{\eta}), P(\bar{\gamma})]} \bar{\lambda}-i_{P(\bar{\lambda})} d\lfloor\bar{\eta}, \bar{\lambda}\rfloor+\mathcal{L}_{P(\bar{\gamma})}\lfloor\bar{\eta}, \bar{\lambda}\rfloor-i_{[P(\bar{\eta}), P(\bar{\lambda})]} d \bar{\gamma} \\
= & \mathcal{L}_{P(\bar{\eta})} \mathcal{L}_{P(\bar{\gamma})} \bar{\lambda}-i_{P(\bar{\lambda})} \mathcal{L}_{P(\bar{\eta})} d \bar{\gamma}+i_{P(\bar{\lambda})} \mathcal{L}_{P(\bar{\gamma})} d \bar{\eta}-\mathcal{L}_{P(\bar{\gamma})} i_{P(\lambda)} d \bar{\eta}-i_{[P(\bar{\eta}), P(\bar{\lambda})]} d \bar{\gamma} \\
= & \mathcal{L}_{P(\bar{\eta})} \mathcal{L}_{P(\bar{\gamma})} \bar{\lambda}-\mathcal{L}_{P(\bar{\eta})} i_{P(\bar{\lambda})} d \bar{\gamma}+i_{P(\bar{\lambda})} \mathcal{L}_{P(\bar{\gamma})} d \bar{\eta}-\mathcal{L}_{P(\bar{\gamma})} i_{P(\bar{\lambda})} d \bar{\eta} \\
= & \mathcal{L}_{P(\bar{\eta})}\lfloor\bar{\gamma}, \bar{\lambda}\rfloor-i_{[P(\bar{\gamma}), P(\bar{\lambda})]} d \bar{\eta}=\lfloor\bar{\eta},\lfloor\bar{\gamma}, \bar{\lambda}\rfloor\rfloor
\end{aligned}
$$

where the second equality holds by $\mathcal{L}_{[X, Y]}=\left[\mathcal{L}_{X}, \mathcal{L}_{Y}\right]$ and the third results from Cartan's magic formula.

It follows from this remark that condition (iii) in Def. 3.1 is equivalent to

(iii)' the space of section $\Gamma(S)$ is closed under the bracket (3.1) and (3.2) holds.

Remark 3.3 (Comparison with [19]). The notion of poly-Poisson structure in Def. 3.1 is slightly more restrictive than the notion introduced by Iglesias, Marrero and Vaquero in [19, Def. 3.1]. The difference is that in [19] our condition (ii) in Def. 3.1, namely $S^{\circ}=\{0\}$, is replaced by the following weaker requirement:

$$
\operatorname{Im}(P) \cap S^{\circ}=\{0\}
$$

We will refer to such objects as weak-poly-Poisson structures.

Let $\left(M_{j}, S_{j}, P_{j}\right), j=1,2$, be $k$-poly-Poisson manifolds.

Definition 3.4. A smooth map $f: M_{1} \rightarrow M_{2}$ is called a poly-Poisson morphism if

a) $f^{*} \bar{\eta} \in S_{1}$ for all $\bar{\eta} \in S_{2}$,

b) for every $x \in M_{1}$ and $\left.\bar{\eta} \in S_{2}\right|_{f(x)},\left.T f\right|_{x}\left(P_{1}\left(\left.T f\right|_{x} ^{*} \bar{\eta}\right)\right)=P_{2}(\bar{\eta})$.

The following are basic examples of Def. 3.1 .

Example 3.1. For $k=1$, a $k$-poly-Poisson structure is simply a usual Poisson structure. Indeed, if $S$ is subbundle of $T^{*} M$, condition (ii) in Def. 3.1 shows that

$$
S=T^{*} M
$$

(Note that this is not guaranteed by the weaker condition (3.3).) Condition (i) shows that $P: T^{*} M \rightarrow T M$ is of the form $P=\pi^{\sharp}$ for a bivector field $\pi \in \Gamma\left(\wedge^{2} T M\right)$, where $\pi^{\sharp}(\alpha)=i_{\alpha} \pi$. Finally, condition (iii) amounts to the usual integrability condition $[\pi, \pi]=0$ (i.e., the bracket on $C^{\infty}(M)$ given by $(f, g) \mapsto \pi(d f, d g)$ satisfies the Jacobi identity). The Lie algebroid structure on $S=T^{*} M$ is the usual one for Poisson manifolds [34]: the anchor is $\pi^{\sharp}$ and the bracket $[\cdot, \cdot]$ on $\Omega^{1}(M)$ is the one such that $[d f, d g]=d(\pi(d f, d g))$. The notion of morphism in Def. 3.4 also recovers to the usual notion of Poisson morphism. 
Example 3.2. Let $(M, \omega)$ be a $k$-poly-symplectic manifold, and consider the injective bundle map $\omega^{b}: T M \rightarrow \oplus_{(k)} T^{*} M$. We define a subbundle $S_{\omega}$ of $\oplus_{(k)} T^{*} M$ and a bundle map $P_{\omega}: S \rightarrow T M$ as follows:

$$
S_{\omega}:=\operatorname{Im}\left(\omega^{b}\right) \quad \text { and } \quad P_{\omega}\left(i_{X} \omega\right):=X \in T M .
$$

See [19, Prop. 2.3 and Example 3.3]. Note that condition (ii) in Def. 3.1] is equivalent to the non-degeneracy of $\omega$.

Moreover, given $k$-poly-symplectic manifolds $\left(M_{j}, \omega_{j}\right), j=1,2$, a diffeomorphism $f: M_{1} \rightarrow M_{2}$ preserves poly-Poisson structures (as in Def. 3.4) if and only if

$$
f^{*} \omega_{2}=\omega_{1} \text {. }
$$

Example 3.3. Let $Q$ be a manifold. We can always regard it as a Poisson manifold with the Poisson bracket that is identically zero. For each $k$, we can also view $Q$ as a $k$-poly-Poisson manifold, and this can be done in several ways. For example, $S_{1}=\oplus_{(k)} T^{*} Q$ and $P_{1}=0$ define a poly-Poisson structure on $Q$, and the same is true for $S_{2}=\left\{\alpha \oplus \ldots \oplus \alpha \mid \alpha \in T^{*} Q\right\} \subset \oplus_{(k)} T^{*} Q$ and $P_{2}=0$, or $S_{3}=\{\alpha \oplus 0 \oplus \ldots \oplus 0 \mid \alpha \in$ $\left.T^{*} Q\right\} \subset \oplus_{(k)} T^{*} Q$ and $P_{3}=0$.

Considering $\oplus_{(k)} T^{*} Q$ equipped with its poly-symplectic structure (see Example 2.1), the natural projection $\oplus_{(k)} T^{*} Q \rightarrow Q$ is a poly-Poisson map when $Q$ is equipped with either one of the poly-Poisson structures $\left(S_{i}, P_{i}\right)$, for $i=1,2,3$.

Remark 3.5. It is a well-known fact in Poisson geometry that $M$ is a Poisson manifold and $f: M \rightarrow N$ is a surjective submersion, then there is at most one Poisson structure on $N$ for which $f$ is a Poisson map. Example 3.3 shows that this is not necessarily the case for $k$-poly-Poisson structures, for $k \geq 2$.

On the other hand, let $M$ be a k-poly-Poisson manifold and $f: M \rightarrow N$ be a surjective submersion. Then if $\left(S_{1}, P_{1}\right)$ and $\left(S_{2}, P_{2}\right)$ are $k$-poly-Poisson structures on $N$ for which $f$ is a poly-Poisson map and we know that $S_{1}=S_{2}$, then $P_{1}=P_{2}$.

As explained in [19, Example 3.8], the product of Poisson manifolds carries a natural poly-Poisson structure.

Example 3.4. Let $\left(M_{j}, \pi_{j}\right), j=1, \ldots, k$, be Poisson manifolds. Let $M=M_{1} \times \ldots \times$ $M_{k}$. Denote by $S_{j}$ the natural inclusion of $T^{*} M_{j}$ into $T^{*} M$, and let $S \subset \oplus_{(k)} T^{*} M$ be defined by $S:=S_{1} \oplus \ldots \oplus S_{k}$. Consider the bundle map $P: S \rightarrow T M$,

$$
P\left(\alpha_{1}, \ldots, \alpha_{k}\right)=\left(\pi_{1}^{\sharp}\left(\alpha_{1}\right), \ldots, \pi_{k}^{\sharp}\left(\alpha_{k}\right)\right),
$$

where $\alpha_{j} \in S_{j}$. One may verify that $(M, S, P)$ is a $k$-poly-Poisson manifold directly from the definition.

In addition, let $f_{j}:\left(M_{j}, \pi_{j}\right) \rightarrow\left(N_{j}, \Lambda_{j}\right)$ for $j=1, \ldots, k$, be $k$ Poisson maps between the Poisson manifolds $M_{j}$ and $N_{j}$ respectively. From the construction above we obtain $k$-poly-Poisson structures $\left(S_{M}, P_{M}\right)$ and $\left(S_{N}, P_{N}\right)$ on the product manifolds $M=\prod_{j=1}^{k} M_{j}$ and $N=\prod_{j=1}^{k} N_{j}$, and denote by $p_{j}^{M}: M \rightarrow M_{j}$, and $p r_{j}^{N}: N \rightarrow N_{j}$ the natural projections. The Poisson maps $f_{j}$ induce a product map $\bar{f}=\left(f_{1}, \ldots, f_{k}\right): M \rightarrow N$ that, as a consequence of the definition of the $k$-poly-Poisson manifold and the relations $\mathrm{pr}_{j}^{N} \circ \bar{f}=f_{j} \circ p r_{j}^{M}$, is a poly-Poisson map.

The next example is a particular case of the direct-sum of linear Poisson structures treated in [19, Example 3.9]. 
Example 3.5. Let $\mathfrak{g}$ be a Lie algebra, and let

$$
\mathfrak{g}_{(k)}:=\mathfrak{g} \times \stackrel{(k}{\cdots} \times \mathfrak{g}, \quad \mathfrak{g}_{(k)}^{*}:=\mathfrak{g}^{*} \times \stackrel{(k}{*} \times \mathfrak{g}^{*} .
$$

For $u \in \mathfrak{g}$, let $u_{j} \in \mathfrak{g}_{(k)}$ denote the element $(0, \ldots, 0, u, 0, \ldots, 0)$, with $u$ in the $j$-th entry. Since $\mathfrak{g}^{*}$ is equipped with its Lie-Poisson structure, $\mathfrak{g}_{(k)}^{*}$ naturally carries a product poly-Poisson structure, as in Example 3.4. More important to us is the following direct-sum poly-Poisson structure [19] : over each $\zeta=\left(\zeta_{1}, \ldots, \zeta_{k}\right) \in \mathfrak{g}_{(k)}^{*}$, we define

$$
\left.S\right|_{\zeta}:=\left\{\left(u_{1}, \ldots, u_{k}\right) \mid u \in \mathfrak{g}\right\} \subseteq \oplus_{(k)} T_{\zeta}^{*} \mathfrak{g}_{(k)}^{*} \cong \oplus_{(k)} \mathfrak{g}_{(k)},
$$

and the bundle map $P: S \rightarrow T \mathfrak{g}_{(k)}^{*}$,

$$
P_{\zeta}\left(u_{1}, \ldots, u_{k}\right):=\left(\operatorname{ad}_{u}^{*} \zeta_{1}, \ldots, \operatorname{ad}_{u}^{*} \zeta_{k}\right) \in T_{\zeta} \mathfrak{g}_{(k)}^{*} \cong \mathfrak{g}_{(k)}^{*} .
$$

We remark that $S$ satisfies (ii) in Def. 3.1, not just (3.3).

3.2. Poly-Poisson structures and poly-symplectic groupoids. We will now justify our definition of poly-Poisson structure in Def. 3.1 in light of its relation with poly-symplectic groupoids.

Let $(M, S, P)$ be a $k$-poly-Poisson manifold. We saw in Section 3.1 that the vector subbundle $S \subseteq \oplus_{(k)} T^{*} M$ is a Lie algebroid, with anchor $P: S \rightarrow T M$ and bracket (3.1).

Lemma 3.6. Let $\mu: S \hookrightarrow \oplus_{(k)} T^{*} M$ be the inclusion. Then $\mu$ is an IM polysymplectic form on the Lie algebroid $S \rightarrow M$, i.e., $\mu$ satisfies (2.6), (2.7) and (2.11).

Conversely, any IM poly-symplectic form $\mu: A \rightarrow \oplus_{(k)} T^{*} M$ is equivalent to one coming from a $k$-poly-Poisson structure.

Proof. Note that (2.6) is just (i) in Def. 3.1, while property (2.7) follows from (iii) in Def. 3.1, Since $\mu$ is an inclusion, $\operatorname{ker}(\mu)=\{0\}$. The second condition in (2.11) is (ii) in Def. 3.1.

On the other hand, given an IM poly-symplectic form $\mu: A \rightarrow \oplus_{(k)} T^{*} M$, we define $S=\operatorname{Im}(\mu)$. Note (from the first condition in (2.11)) that $\mu$ is a vector-bundle isomorphism onto $S$, and let $P: S \rightarrow T M$ be its inverse $S \rightarrow A$ composed with the anchor $A \rightarrow T M$. One may directly verify from conditions (2.6), (2.7) and (2.11) that $S$ and $P$ define a $k$-poly-Poisson structure, and that $\mu$ is equivalent to the inclusion $S \hookrightarrow \oplus_{(k)} T^{*} M$.

In short, the lemma says that a $k$-poly-Poisson manifold $(M, S, P)$ endows $S$ with a Lie algebroid structure for which the inclusion $S \hookrightarrow \oplus_{(k)} T^{*} M$ is an IM polysymplectic form, and that any IM poly-symplectic form is equivalent to one of this type.

Following Corollary 2.5, we see that poly-Poisson manifolds are the infinitesimal counterparts of poly-symplectic groupoids, as explained by the next result. For a $k$-poly symplectic groupoid $(\mathcal{G} \rightrightarrows M, \omega)$, let $\mu: A \rightarrow \oplus_{(k)} T^{*} M$ be the bundle map determined by $\omega$ as in Cor. 2.5. Explicitly, using the natural decomposition $\left.T \mathcal{G}\right|_{M}=T M \oplus A$,

for $u \in A$.

$$
\mu(u)=\left.\omega^{b}(u)\right|_{\oplus_{(k)} T M},
$$


Theorem 3.7 (Integration of poly-Poisson structures). If ( $\mathcal{G} \rightrightarrows M, \omega)$ is a k-polysymplectic groupoid, then there exists a unique $k$-poly-Poisson structure $(S, P)$ on $M$ such that $S=\operatorname{Im}(\mu)$ while $P$ is determined by the fact that the target map $t: \mathcal{G} \rightarrow M$ is a poly-Poisson morphism.

Conversely, let $(M, S, P)$ be a k-poly-Poisson manifold and $\mathcal{G} \rightrightarrows M$ be a sourcesimply-connected groupoid integrating the Lie algebroid $S \rightarrow M$. Then there is a $\omega \in \Omega^{2}\left(\mathcal{G}, \mathbb{R}^{k}\right)$, unique up to isomorphism, making $\mathcal{G}$ into a poly-symplectic groupoid for which $t: \mathcal{G} \rightarrow M$ is a poly-Poisson morphism.

We say that a poly-symplectic groupoid integrates a poly-Poisosn structure if they are related as in the theorem. We observe that this correspondence between sourcesimply-connected poly-symplectic groupoids and poly-Poisson manifolds (with integrable Lie algebroid) extends the well-known relationship between symplectic groupoids and Poisson manifolds when $k=1$, see [11, 26].

Proof. We know from Corollary 2.5 that multiplicative poly-symplectic structures on $\mathcal{G} \rightrightarrows M$ correspond to IM poly-symplectic forms $\mu$ on its Lie algebroid $A \rightarrow M$ via

$$
i_{u R} \omega=t^{*}(\mu(u)),
$$

and that $\mu$ corresponds to a poly-Poisson structure $(S, P)$ on $M$, as described in Lemma 3.6. It remains to verify that condition (3.5) implies that $t$ is a poly-Poisson map.

Let $\alpha \in \operatorname{Im}(\mu)=S$. Then $\alpha=\mu(u)$ for a unique $u \in A$, and $P(\alpha)=\rho(u)$. Let $\left(S_{\omega}, P_{\omega}\right)$ be the poly-Poisson structure defined by $\omega$, as in (3.4). Then (3.5) says that $t^{*} \alpha \in S_{\omega}$ and $u^{R}=P_{\omega}\left(t^{*} \alpha\right)$; the fact that on any Lie groupoid we have $t_{*}\left(u^{R}\right)=\rho(u)$ implies that $t_{*} P_{\omega}\left(t^{*} \alpha\right)=P(\alpha)$, i.e., $t$ is a poly-Poisson map.

Remark 3.8. Given a k-poly-symplectic groupoid $(\mathcal{G} \rightrightarrows M, \omega)$, the uniqueness of the induced poly-Poisson structure $(S, P)$ on $M$ follows from Remark 3.5: note that $S$ is determined by $\omega$, while $P$ is completely defined from the property that $t$ is a poly-Poisson map.

We illustrate the correspondence in Theorem 3.7 with some simple examples.

Example 3.6. The $k$-poly-symplectic manifold $\oplus_{(k)} T^{*} Q$ of Example 2.1 is a polysymplectic groupoid over $Q$, with respect to fibrewise addition; the source and target maps coincide with the projection $\oplus_{(k)} T^{*} Q \rightarrow Q$. The corresponding $k$-poly-Poisson structure on $Q$ is the trivial one, given by $S:=\oplus_{(k)} T^{*} Q$ and $P=0$. Note that Example 3.3 shows other poly-Poisson structures on $Q$ for which the projection $\oplus_{(k)} T^{*} Q \rightarrow Q$ is a poly-Poisson map, but there is only one with the bundle $S$ prescribed by Theorem 3.7 .

Example 3.7. Let $(M, \omega)$ be a $k$-poly-symplectic manifold, that we view as a polyPoisson manifold as in Example 3.2. The non-degeneracy of $\omega$ implies that the Lie algebroid $\left(S_{\omega}, P_{\omega}\right)$ is isomorphic to $T M$. Hence this poly-Poisson structure is integrated by the pair groupoid $M \times M \rightrightarrows M$, equipped with the $k$-poly-symplectic structure $t^{*} \omega-s^{*} \omega \in \Omega^{2}\left(M \times M, \mathbb{R}^{k}\right)$, where $s, t$ are the source and target maps on the pair groupoid, i.e $t(x, y)=x$ and $s(x, y)=y$.

Example 3.8. Consider Poisson manifolds $\left(M_{j}, \pi_{j}\right), j=1, \ldots, k$, and equip $M=$ $M_{1} \times \ldots \times M_{k}$ with the product poly-Poisson structure of Example 3.4. For each 
$j$, suppose that $\left(\mathcal{G}_{j} \rightrightarrows M_{j}, \omega_{j}\right)$ is a symplectic groupoid integrating $\left(M_{j}, \pi_{j}\right)$. Then the product poly-symplectic groupoid $\mathcal{G}=\mathcal{G}_{1} \times \ldots \times \mathcal{G}_{k}$ of Prop. 2.2 integrates $M$. Indeed, one may verify that the bundle $S$ on $M$ described in Example 3.4 agrees with the one prescribed by Theorem 3.7 and, as a consequence of the construction of poly-Poisson maps as products of Poisson maps in Example 3.4 the target map on $\mathcal{G} \rightrightarrows M$ is a poly-Poisson map.

Example 3.9 (Lie-Poisson structures). Let $\mathbb{G}$ be a Lie group and $\mathfrak{g}$ its Lie algebra. As seen in Example 2.1. $\oplus_{(k)} T^{*} \mathbb{G}$ has a natural $k$-poly-symplectic structure $\omega$.

The diagonal coadjoint action of $\mathbb{G}$ on $\mathfrak{g}_{(k)}^{*}$, denoted by $\operatorname{Ad}_{g}^{*}$, endows $\mathbb{G} \times \mathfrak{g}_{(k)}^{*}$ with a groupoid structure over $\mathfrak{g}_{(k)}^{*}$, with source and target maps given by

$$
s(g, \zeta)=\zeta, \quad t(g, \zeta)=\operatorname{Ad}_{g}^{*} \zeta
$$

and multiplication $m((g, \zeta),(h, \eta))=(g h, \eta)$ if $\operatorname{Ad}_{h}^{*} \eta=\zeta$. Using the identification $T^{*} \mathbb{G} \cong \mathbb{G} \times \mathfrak{g}^{*}$ (by right translation), we see that

$$
\oplus_{(k)} T^{*} \mathbb{G} \cong \mathbb{G} \times \mathfrak{g}_{(k)}^{*},
$$

so we may consider $\oplus_{(k)} T^{*} \mathbb{G}$ as a Lie groupoid, and its poly-symplectic structure $\omega$ makes it into a poly-symplectic groupoid. This structure integrates the direct-sum poly-Poisson structure on $\mathfrak{g}_{(k)}^{*}$ described in Example 3.5. Indeed, $t$ has the Poisson maps $\mathbb{G} \times \mathfrak{g}^{*} \rightarrow \mathfrak{g}^{*}$ as its coordinates, so it is a poly-Poisson map. And one can check that the bundle $S$ of the direct-sum poly-Poisson structure is the one induced by the poly-symplectic structure $\omega$ according to Theorem 3.7.

Remark 3.9. More generally: following [19] there is a direct-sum poly-Poisson structure on $A^{*} \oplus \ldots \oplus A^{*}$ where $A^{*} \rightarrow M$ is endowed with the linear Poisson structures (defined on the dual bundle to the Lie algebroid $A \rightarrow M$ ). Each $A^{*}$ is integrated by the symplectic groupoid $T^{*} \mathcal{G} \rightrightarrows A^{*}$, where $\mathcal{G} \rightrightarrows M$ is the groupoid integrating $A$, and it can be similarly proved that the direct sum $T^{*} \mathcal{G} \oplus \ldots \oplus T^{*} \mathcal{G}$ over $\mathcal{G}$ is the poly-symplectic groupoid integrating $A^{*} \oplus \ldots \oplus A^{*}$.

3.3. Poly-symplectic foliation. It is well known that any Poisson manifold has an underlying symplectic foliation which uniquely determines the Poisson structure. More generally, let $(S, P)$ be a $k$-poly-Poisson structure on $M$. Since $S$ has a Lie algebroid structure, the distribution $D:=P(S) \subseteq T M$ is integrable, and its leaves define a singular foliation on $M$. Each leaf $\iota: \mathcal{O} \hookrightarrow M$ carries an $\mathbb{R}^{k}$-valued 2-form $\omega_{\mathcal{O}}$ determined by the condition

$$
\omega_{\mathcal{O}}^{b}: T \mathcal{O} \rightarrow \oplus_{(k)} T^{*} \mathcal{O}, \quad P(\bar{\eta}) \mapsto \iota^{*} \bar{\eta} .
$$

The fact that the 2 -form $\omega_{\mathcal{O}}$ on $\mathcal{O}$ is well defined follows from (i) in Def. 3.1. (ii) guarantees that it is non-degenerate and (iii) that it is closed, see [19, Sec. 3]. So $(S, P)$ determines a singular foliation on $M$ with $(k+1)$-poly-symplectic leaves.

A first remark on the poly-symplectic foliation of a $k$-poly-Poisson structure is that, in contrast with the case $k=1$, different $k$-poly-Poisson structures may correspond to the same poly-symplectic foliation, as shown in the next example.

Example 3.10. Let $\omega_{t}$ be a smooth family of $k$-poly-symplectic forms on $M$ parametrized by $t \in \mathbb{R}$ and define the following vector subbundles of $\oplus_{(k)} T^{*}(M \times \mathbb{R})$ : 


$$
\begin{aligned}
& \left.S_{1}\right|_{(m, t)}:=\left\{\left(i_{X} \omega_{t}, r_{1} \oplus \cdots \oplus r_{k}\right) \mid X \in T_{m} M, r_{j} \in T_{t}^{*} \mathbb{R}\right\}, \\
& \left.S_{2}\right|_{(m, t)}:=\left\{\left(i_{X} \omega_{t}, r \oplus \cdots \oplus r\right) \mid X \in T_{m} M, r \in T_{t}^{*} \mathbb{R}\right\}, \\
& \left.S_{3}\right|_{(m, t)}:=\left\{\left(i_{X} \omega_{t}, r \oplus 0 \cdots \oplus 0\right) \mid X \in T_{m} M, r \in T_{t}^{*} \mathbb{R}\right\} ;
\end{aligned}
$$

on each $S_{j}$ we define $P_{j}\left(i_{X} \omega_{t}, \bar{\gamma}\right)=X$. Observe that each $\left(S_{j}, P_{j}\right)$ is a poly-Poisson structure on $M \times \mathbb{R}$ but these three $k$-poly-Poisson structures have the same polysymplectic foliation on $M \times \mathbb{R}$. Same conclusion holds for the weak-poly-Poisson structure given by

$$
\left.S_{0}\right|_{(m, t)}:=\left\{\left(i_{X} \omega_{t}, 0\right) \mid X \in T_{m} M\right\} \text { and } P_{0}\left(i_{X} \omega_{t}, 0\right)=X
$$

where the poly-symplectic foliation is described on Theorem 3.4 on [19].

We now discuss the possibility of defining a poly-Poisson structure from a polysymplectic foliation. Given a subspace $D_{m} \subseteq T_{m} M$, for $m \in M$, equipped with a $(k+1)$-poly-symplectic form $\omega_{m}$, we consider the subspace $S_{m} \subseteq \oplus_{(k)} T^{*} M$ given by

$$
S_{m}:=\left\{\bar{\eta} \in \oplus_{(k)} T_{m}^{*} M\left|\exists X \in D_{m}, \bar{\eta}\right|_{\oplus_{(k)} D_{m}}=i_{X} \omega_{m}\right\},
$$

which has dimension $k(n-p)+p$, where $p$ is the dimension of $D_{m}$. One may verify that $S_{m}^{\circ}=\{0\}$ and there is a well-defined map $P_{m}: S_{m} \rightarrow D_{m} \subseteq T_{m} M$,

$$
P_{m}(\bar{\eta})=X \text {, where }\left.\bar{\eta}\right|_{\oplus_{(k)} D_{m}}=i_{X} \omega_{m}
$$

Given now a regular poly-symplectic foliation on $M$, letting $D$ be its tangent distribution, we use the previous pointwise construction to see that (3.7) defines a subbundle $S \subseteq \oplus_{(k)} T^{*} M$, satisfying $S^{\circ}=\{0\}$, and equipped with a bundle map $P: S \rightarrow T M$. Moreover, using the fact that the $\mathbb{R}^{k}$-valued form defined on each leaf is closed, it follows that $(S, P)$ satisfies (iii) in Def. 3.1, so it is a poly-Poisson structure. In conclusion we have the following proposition (see [19, Sec. 3]),

Proposition 3.10. If $(D, \omega)$ is a regular $k$-poly-symplectic foliation on $M$ then $(S, P)$, defined pointwise by (3.7) and (3.8), is a k-poly-Poisson on $M$.

In particular, if the regular $k$-poly-symplectic foliation on $M$ comes from a weakpoly-Poisson structure as in [19, Theorem 3.4], then the poly-Poisson structure on the proposition is an "extension" of the weak-poly-Poisson structure In order to illustrate last claim and the poly-Poisson structure from (3.7) and (3.8) we apply the proposition to the regular poly-symplectic foliation given in Example 3.10, which is the same for each poly-Poisson strucutres $\left(S_{j}, P_{j}\right)$ for $j=1,2,3$ and for the weakpoly-Poisson $\left(S_{0}, P_{0}\right)$, and get the "maximal" poly-Poisson structure $\left(S_{1}, P_{1}\right)$.

3.4. Relation with AV-Dirac structures. It is well known that Poisson structures on $M$ can be understood as special types of Dirac structures in the Courant algebroid $T M \oplus T^{*} M[12$. As we now see, this picture can be generalized to polyPoisson structures. We consider the bundle $\mathbb{A}:=T M \oplus\left(\oplus_{(k)} T^{*} M\right)$, equipped with the $\left(\mathbb{R}^{k}\right.$-valued) fibrewise inner product

$$
\langle X \oplus \bar{\eta}, Y \oplus \bar{\gamma}\rangle:=i_{X} \bar{\gamma}+i_{Y} \bar{\eta}
$$

and bracket on sections of $\mathbb{A}$ given by

$$
\llbracket X \oplus \bar{\eta}, Y \oplus \bar{\gamma} \rrbracket:=[X, Y] \oplus \mathcal{L}_{X} \bar{\gamma}-i_{Y} d \bar{\eta} .
$$


For $k=1$, this is the standard Courant algebroid $T M \oplus T^{*} M$. In general, this is a very particular case of the $A V$-Courant algebroids introduced in [24, Sec. 2] (with respect to the Lie algebroid $A=T M$ and representation on $V=M \times \mathbb{R}^{k} \rightarrow M$ given by the Lie derivative $\mathcal{L}_{X}\left(f_{1}, \ldots, f_{k}\right)=\left(\mathcal{L}_{X} f_{1}, \ldots, \mathcal{L}_{X} f_{k}\right)$ on $\left.C^{\infty}\left(M, \mathbb{R}^{k}\right)\right)$.

Following [24], one may consider $A V$-Dirac structures on any AV-Courant algebroid: these are subbundles $L \subseteq \mathbb{A}$ which are lagrangian, i.e.,

$$
L=L^{\perp},
$$

with respect to the fibrewise inner product, and which are involutive with respect to the bracket $\llbracket \cdot, \cdot \rrbracket$ on $\Gamma(\mathbb{A})$. Recall that $L$ is called isotropic if $L \subset L^{\perp}$.

Example 3.11. Any $k$-poly-symplectic structure $\omega$ on $M$ may be seen as an AVDirac structure in $\mathbb{A}:=T M \oplus\left(\oplus_{(k)} T^{*} M\right)$ via

$$
L:=\operatorname{graph}(\omega)=\left\{X \oplus i_{X} \omega \mid X \in T M\right\} .
$$

Note that this $L$ satisfies the additional condition

$$
L \cap\left(\oplus_{(k)} T^{*} M\right)=\{0\} .
$$

In fact, poly-symplectic structures on $M$ are in one-to-one correspondence with AVDirac structures which project isomorphically over $T M$ and satisfy $L \cap T M=\{0\}$ and (3.10).

Our goal now is to define, in the same way, a subbundle $L$ from a poly-Poisson structure $(S, P)$, i.e. consider

$$
L=\{P(\bar{\eta}) \oplus \bar{\eta} \mid \bar{\eta} \in S\} .
$$

Note that $L$ is isotropic as a consequence of (i) in Definition 3.1, But, as we now see, the lagrangian condition generally fails.

Example 3.12. Let $\mathfrak{g}$ be a Lie algebra and consider the poly-Poisson structure on $\mathfrak{g}_{(2)}^{*}$ as in Example 3.5. Observe that $L$ over the point $\zeta=(0,0) \in \mathfrak{g}_{(2)}^{*}$ can be written as

$$
L_{\zeta}=\{(0,0) \oplus((u, 0),(0, u)) \mid u \in \mathfrak{g}\} .
$$

But for any $v_{1}, v_{2}, w_{1}, w_{2} \in \mathfrak{g}$ we have $(0,0) \oplus\left(\left(v_{1}, v_{2}\right),\left(w_{1}, w_{2}\right)\right) \in L^{\perp}$, hence $L$ is properly contained in $L^{\perp}$.

Therefore, in general, poly-Poisson structures are not AV-Dirac structures. In order to include poly-Poisson structures in the formalism of AV-Courant algebroids, one then needs to relax the lagrangian condition (3.9).

Let us consider subbundles $L \subseteq T M \oplus\left(\oplus_{(k)} T^{*} M\right)$ satisfying

$$
L=L^{\perp} \cap(L+T M) .
$$

Note that (3.9) implies that (3.11) holds, but the converse is not true.

The following results characterize $k$-poly-Poisson structures as subbundles of $\mathbb{A}=$ $T M \oplus\left(\oplus_{(k)} T^{*} M\right):$

Proposition 3.11. There is a one-to-one correspondence among the following:

(a) k-poly-Poisson structures $(S, P)$ on $M$,

(b) Involutive, isotropic subbundles $L \subset \mathbb{A}$ satisfying $L^{\perp} \cap T M=\{0\}$,

(c) Involutive subbundles $L \subset \mathbb{A}$ satisfying (3.11) and $L \cap T M=\{0\}$. 
Proof. Given a $k$-poly-Poisson structure $(S, P)$, we define the subbundle $L \subset \mathbb{A}$ by

$$
L=\{P(\bar{\eta}) \oplus \bar{\eta} \mid \bar{\eta} \in S\} .
$$

This bundle is isotropic by condition (i) in Def. 3.1, condition (ii) amounts to $L^{\perp} \cap$ $T M=\{0\}$ while (iii) is equivalent to the involutivity of $L$. Conversely, given $L$ as in (b), the image of the natural projection $L \rightarrow \oplus_{(k)} T^{*} M$ defines a vector bundle $S$ and a bundle map $P: S \rightarrow T M$ by

$$
P(\bar{\eta})=X \text { if and only if } X \oplus \bar{\eta} \in L,
$$

in such a way that $(S, P)$ is a $k$-poly-Poisson structure. This gives the correspondence between (a) and (b).

For a $k$-poly-Poisson structure $(S, P)$ and $L$ as in (3.12), one may directly verify that (i) in Def. 3.1 implies that (3.11) holds, while (ii) implies that $L \cap T M=\{0\}$, so $L$ satisfies the properties in (c). It remains to check that given an $L$ as in (c), then it satisfies the properties described in (b). Note that (3.11) implies that $L$ is isotropic and that $L \cap T M=L^{\perp} \cap T M$, so that $L^{\perp} \cap T M=\{0\}$.

Remark 3.12. For $k=1$, the objects in (b) and (c) are just usual Dirac structures on $M$, satisfying the additional condition $L \cap T M=\{0\}$ (conditions (3.9) and (3.11) turn out to be equivalent for $k=1$ ), while the objects in (a) are usual Poisson structures. So for $k=1$ Prop. 3.11 boils down to the known characterization of Poisson structures as particular types of Dirac structures.

\section{Symmetries And ReduCtion}

We now discuss poly-Poisson structures and poly-symplectic groupoids in the presence of symmetries, with the aim of using reduction as a tool for integration of poly-Poisson manifolds, along the lines of [30, 17].

4.1. Poly-Poisson actions. An action $\varphi$ of a Lie group $\mathbb{G}$ on a $k$-poly-Poisson manifold $(M, S, P)$ is a poly-Poisson action if for each $g \in \mathbb{G}$ the diffeomorphism $\varphi_{g}: M \rightarrow M$ is a poly-Poisson morphism (Def. 3.4). In the case of $k$-poly-symplectic manifold $(M, \omega)$, this means that $\varphi_{g}^{*} \omega=\omega$, see Example 3.2.

Let us consider a poly-Poisson action $\varphi$ of a Lie group $\mathbb{G}$ on $(M, S, P)$, and let us assume henceforth that this action is free and proper, so that we have a principal G-bundle:

$$
\Pi: M \rightarrow M / \mathbb{G}
$$

Let $V \subseteq T M$ denote the vertical bundle defined by this action.

It is well-known that, when $k=1$, i.e., $M$ is an ordinary Poisson manifold, $M / \mathbb{G}$ inherits a Poisson structure for which $\Pi$ is a Poisson map. For poly-Poisson manifolds, we will need additional conditions. We call the action $\varphi$ is reducible if

$$
\left\{\begin{array}{l}
\text { (a) } S \cap \oplus_{k} \operatorname{Ann}(V) \text { has constant rank, } \\
\text { (b) }\left(S \cap \oplus_{k} \operatorname{Ann}(V)\right)^{\circ} \subset V .
\end{array}\right.
$$


The projection map (4.1) induces a map $d \Pi_{(k)}: \oplus_{(k)} T M \rightarrow \Pi^{*}\left(\oplus_{(k)} T(M / \mathbb{G})\right)$, and its transpose is an injective bundle map $\Pi^{*}\left(\oplus_{(k)} T^{*}(M / \mathbb{G})\right) \rightarrow \oplus_{(k)} T^{*} M$, whose image is the subbundle $\oplus_{(k)} \operatorname{Ann}(V) \subseteq \oplus_{(k)} T^{*} M$. So we have an induced isomorphism

$$
\Pi^{*}\left(\oplus_{(k)} T^{*}(M / \mathbb{G})\right) \stackrel{\sim}{\rightarrow} \oplus_{(k)} \operatorname{Ann}(V) .
$$

The next result is analogous to [19, Thm. 4.1] (but stated for our stronger notion of poly-Poisson structure).

Theorem 4.1. Let us consider a poly-Poisson $\mathbb{G}$-action on a $k$-poly-Poisson manifold $(M, S, P)$ which is free and proper, and reducible. Then $M / \mathbb{G}$ inherits a $k$-polyPoisson structure $\left(S_{r e d}, P_{\text {red }}\right)$, where the subbundle $S_{\text {red }} \subseteq \oplus_{(k)} T^{*}(M / \mathbb{G})$ corresponds to $S \cap \oplus_{(k)} \operatorname{Ann}(V)$ via (4.3), and $P_{\text {red }}$ is unique so that the quotient map (4.1) is a k-poly-Poisson morphism.

Proof. The first condition in (4.2) guarantees that $S_{\text {red }} \subseteq \oplus_{(k)} T^{*}(M / \mathbb{G})$, defined by the condition that $\Pi^{*} S_{r e d}$ is isomorphic to $S \cap \oplus_{(k)} \operatorname{Ann}(V)$ under (4.3), is a vector subbundle. Note that we have a natural map $\Pi^{*}\left(S_{r e d}\right) \rightarrow \Pi^{*}(T(M / \mathbb{G}))$ given by the composition

$$
\Pi^{*}\left(S_{r e d}\right) \stackrel{d \Pi_{(k)}^{*}}{\longrightarrow} S \cap \oplus_{(k)} \operatorname{Ann}(V) \stackrel{P}{\longrightarrow} T M \stackrel{d \Pi}{\longrightarrow} \Pi^{*}(T(M / \mathbb{G})),
$$

and this defines a bundle map

$$
P_{\text {red }}: S_{\text {red }} \rightarrow T(M / \mathbb{G})
$$

as a consequence of the $\mathbb{G}$-invariance of $(S, P)$.

To check that $\left(S_{\text {red }}, P_{\text {red }}\right)$ defines a $k$-poly-Poisson structure on $M / \mathbb{G}$, one must verify that it satisfies conditions (i), (ii), and (iii) in Def. 3.1. Condition (i) follows directly from the definition of $\left(S_{\text {red }}, P_{\text {red }}\right)$ and the fact that this condition is satisfied by $(S, P)$. It is also routine to check that condition (iii) holds for $\left(S_{\text {red }}, P_{\text {red }}\right)$, given that it holds for $(S, P)$.

As for condition (ii), it is a consequence of property (b) in (4.2). Indeed, by the way $S_{\text {red }}$ is defined, the fact that $\bar{X} \in S_{\text {red }}^{\circ}$ implies that $\bar{X}=d \Pi(X)$, for $X \in$ $\left(S \cap \oplus_{(k)} \operatorname{Ann}(V)\right)^{\circ}$. But then (b) in (4.2) implies that $\bar{X}=d \Pi(X)=0$.

It is also clear from the definition of $P_{\text {red }}$ that $\Pi$ is a poly-Poisson map.

We mention two concrete examples, discussed in [19].

\section{Example 4.1.}

(a) Let $Q$ be a manifold equipped with a free and proper $\mathbb{G}$-action, and let $\left(M=\oplus_{(k)} T^{*} Q, \omega\right)$ be the poly-symplectic manifold of Example 2.1. We keep the notation $\operatorname{pr}_{j}: M \rightarrow T^{*} Q$ for the natural projection onto the $j$ thfactor. The cotangent lift of the $\mathbb{G}$-action on $Q$ defines an action on $T^{*} Q$, which induces a $\mathbb{G}$-action on $M$ which preserves the poly-symplectic structure (i.e., it is a poly-Poisson action), and there is a natural identification

$$
M / \mathbb{G} \cong \oplus_{(k)}\left(T^{*} Q / \mathbb{G}\right) .
$$

We observe here that both conditions in (4.2) hold, i.e., the $\mathbb{G}$-action on $M$ is reducible. To verify this fact, let $V \subseteq T M$ be the vertical bundle of the $\mathbb{G}$-action on $M$, so that $V_{j}=d \operatorname{pr}_{j}(V) \subseteq T\left(T^{*} Q\right)$ is the vertical bundle of the 
$\mathbb{G}$-action on the $j$ th-factor $T^{*} Q$. Note that the natural projection $T^{*} Q \rightarrow Q$ induces a projection of $V_{j}^{\omega_{c a n}}$ onto $T Q$, and one then sees that

$$
V_{1}^{\omega_{\text {can }}} \times_{T Q} \ldots \times_{T Q} V_{k}^{\omega_{\text {can }}} \subseteq T\left(T^{*} Q\right) \times_{T Q} \ldots \times_{T Q} T\left(T^{*} Q\right)=T M
$$

is a vector subbundle, that we denote by $W$. One can now check that

$$
S_{\omega} \cap \oplus_{(k)} \operatorname{Ann}(V)=\left\{i_{X} \omega \mid X \in W\right\},
$$

from where one concludes that condition (a) of (4.2) holds. From (4.6), one directly sees that

$$
\begin{aligned}
\left(S_{\omega} \cap \oplus_{(k)} \operatorname{Ann}(V)\right)^{\circ} & =\left(V_{1}^{\omega_{c a n}}\right)^{\omega_{c a n}} \times_{T Q} \ldots \times_{T Q}\left(V_{k}^{\omega_{c a n}}\right)^{\omega_{c a n}} \\
& =V_{1} \times_{T Q} \ldots \times_{T Q} V_{k}=V,
\end{aligned}
$$

showing that (b) of (4.2) also holds. So the action is reducible. As shown in [19, Ex. 4.3], the reduced poly-Poisson structure on $\oplus_{(k)}\left(T^{*} Q / \mathbb{G}\right)$ is the one defined by direct-sum of the natural linear Poisson structure on $T^{*} Q / \mathbb{G}$ (dual to the Atiyah algebroid $T Q / \mathbb{G}$ of the principal bundle $Q \rightarrow Q / \mathbb{G}$ ).

(b) In the particular case of $Q=\mathbb{G}$ with the action by left multiplication, as shown in [19, Ex. 4.2], the poly-Poisson reduction of $\oplus_{(k)} T^{*} \mathbb{G}$ with respect to the lifted $\mathbb{G}$-action is identified with $\mathfrak{g}_{(k)}^{*}$ of Example 3.5.

4.2. Hamiltonian actions on poly-symplectic manifolds. We now consider poly-Poisson actions on poly-symplectic manifolds in the presence of moment maps.

Let $(M, \omega)$ be a $k$-poly-symplectic manifold equipped with a poly-Poisson action of $\mathbb{G}$, denoted by $\varphi$. Consider the diagonal coadjoint action of $\mathbb{G}$ on the space $\mathfrak{g}_{(k)}^{*}$. This action is called hamiltonian [18, 27] if there is a moment map, i.e., a map $J: M \rightarrow \mathfrak{g}_{(k)}^{*}$ that satisfies

$$
\text { (i) } J \circ \varphi_{g}=\operatorname{Ad}_{g}^{*} \circ J \text { and (ii) } i_{u_{M}} \omega=d\langle J, u\rangle \text {. }
$$

for all $u \in \mathfrak{g}$. Here $u_{M} \in \mathfrak{X}(M)$ denotes the infinitesimal generator corresponding to $u \in \mathfrak{g}$.

Example 4.2. Let $(M, \omega)$ be a poly-symplectic manifold such that $\omega=-d \theta$, and assume that $\mathbb{G}$ acts on $M$ preserving the 1 -form $\theta$. Then the maps $J_{1}, \ldots J_{k}: M \rightarrow \mathfrak{g}^{*}$ defined by $\left\langle J_{l}, u\right\rangle=\theta_{l}\left(u_{M}\right), u \in \mathfrak{g}$, define a moment map for the action.

A particular case of this example is when $M=\oplus_{(k)} T^{*} Q$ (as in Example 2.1) and the action of $\mathbb{G}$ on $M$ is the lift of an action on $Q$, see Example 4.1(a). Here the moment map is $\langle J(\bar{\eta}), u\rangle=\left(\left\langle\eta_{j}, u_{Q}\right\rangle\right)_{j=1, \ldots, k}$.

The following observation generalizes a well-known fact in Poisson geometry. Consider $\mathfrak{g}_{(k)}^{*}$ with the poly-Poisson structure of Example 3.5.

Proposition 4.2. The moment map $J: M \rightarrow \mathfrak{g}_{(k)}^{*}$ of a hamiltonian action of $\mathbb{G}$ on $(M, \omega)$ is a poly-Poisson morphism.

Proof. Denote the poly-Poisson structure on $\mathfrak{g}_{(k)}^{*}$ by $(S, P)$, as in Example 3.5. Consider $\left.\left(u_{1}, \ldots, u_{k}\right) \in S\right|_{\zeta}$, and $Y \in T_{x} M$ with $J(x)=\zeta$. By condition (ii) in (4.7) we have

$$
\begin{aligned}
\left(J^{*}\left(u_{1}, \ldots, u_{k}\right)\right)(Y) & =\left(u_{1}, \ldots, u_{k}\right)(d J(Y))=\left(\left\langle d J(Y), u_{j}\right\rangle\right)_{j=1, \ldots, k} \\
& =\left(\left\langle d J_{j}(Y), u\right\rangle\right)_{j=1, \ldots, k}=\left.\left(i_{u_{M}} \omega\right)\right|_{x}(Y),
\end{aligned}
$$


hence $\left(J^{*}\left(u_{1}, \ldots, u_{k}\right)\right)=\left.\left.i_{u_{M}} \omega\right|_{x} \in \operatorname{Im}\left(\omega^{b}\right)\right|_{x}$.

Recall the bundle maps of the poly-Poisson structures on $M$ and $\mathfrak{g}_{(k)}^{*}$ :

$$
\begin{gathered}
\left.P_{\omega}\left(J^{*}\left(u_{1}, \ldots, u_{k}\right)\right)\right|_{x}=P_{\omega}\left(\left.i_{u_{M}} \omega\right|_{x}\right)=\left.u_{M}\right|_{x}, \\
\left.P\right|_{\zeta}\left(u_{1}, \ldots, u_{k}\right)=\left(\operatorname{ad}_{u}^{*} \zeta_{j}\right)_{j=1, \ldots, k}=\left(u_{\mathfrak{g}^{*}}\left(\zeta_{j}\right)\right)_{j=1, \ldots, k} .
\end{gathered}
$$

¿From condition (i) in (4.7) we can derive that $d J_{j}\left(u_{M}(x)\right)=u_{\mathfrak{g}^{*}}\left(\zeta_{j}\right)$, therefore on points $\zeta=J(x)$ we obtain

$$
d J\left(\left.P_{\omega}\right|_{x}\left(J^{*}\left(u_{1}, \ldots, u_{k}\right)\right)=\left(d J_{j}\left(u_{M}(x)\right)\right)=\left(u_{\mathfrak{g}^{*}}\left(\zeta_{j}\right)\right)=\left.P\right|_{\zeta}\left(u_{1}, \ldots, u_{k}\right) .\right.
$$

Let us consider a Hamiltonian $\mathbb{G}$-action on a $k$-poly-symplectic manifold $(M, \omega)$, with moment map $J: M \rightarrow \mathfrak{g}_{(k)}^{*}$. Let $\zeta \in \mathfrak{g}_{(k)}^{*}$ be a clean value for $J$, i.e.,

$$
\left\{\begin{array}{l}
J^{-1}(\zeta) \text { is a submanifold of } M \\
\operatorname{ker}\left(d_{x} J\right)=T_{x} J^{-1}(\zeta), \text { for all } x \in J^{-1}(0) .
\end{array}\right.
$$

The submanifold $J^{-1}(\zeta)$ is invariant by the action of $\mathbb{G}_{\zeta}$, the isotropy group of $\zeta$ with respect to the diagonal coadjoint action. We assume that the $\mathbb{G}_{\zeta}$-action on $J^{-1}(\zeta)$ is free and proper, so we can consider the reduced manifold

$$
M_{\zeta}:=J^{-1}(\zeta) / \mathbb{G}_{\zeta}
$$

We let $\Pi_{\zeta}: J^{-1}(\zeta) \rightarrow M_{\zeta}$ be the natural projection map, and $i_{\zeta}: J^{-1}(\zeta) \rightarrow M$ the inclusion. We denote by $V_{\zeta} \subseteq T J^{-1}(\zeta)$ the vertical bundle with respect to the $\mathbb{G}_{\zeta}$-action. It follows from (i) in (4.7) that $V_{\zeta}=V \cap T J^{-1}(\zeta)$, while (ii) implies that

$$
V_{\zeta} \subseteq \operatorname{ker}\left(i_{\zeta}^{*} \omega\right)
$$

This last condition, together with the $\mathbb{G}_{\zeta}$-invariance of $i_{\zeta}^{*} \omega$, implies that $i_{\zeta}^{*} \omega$ is basic, i.e., there exists a (unique) closed form $\omega_{\text {red }} \in \Omega^{2}\left(M_{\zeta}, \mathbb{R}^{k}\right)$ so that

$$
\Pi_{\zeta}^{*} \omega_{\text {red }}=i_{\zeta}^{*} \omega
$$

In general, however, the form $\omega_{\text {red }}$ fails to be poly-symplectic, as it may be degenerate; indeed, it is nondegenerate if and only if we have an equality in (4.9).

Note that (ii) in (4.7) says that

$$
T J^{-1}(\zeta)=\operatorname{ker}(d J)=V^{\omega}
$$

where, for a subbundle $W \subseteq T M$, we use the notation $W^{\omega}=\{Y \in T M, \mid \omega(X, Y)=$ $0 \forall X \in W\}$. Writing $S=\operatorname{Im}\left(\omega^{b}\right)$, one may also check that

$$
(\operatorname{ker}(d J))^{\omega}=\left(V^{\omega}\right)^{\omega}=\left(S \cap \oplus_{k} \operatorname{Ann}(V)\right)^{\circ},
$$

from where we conclude that

$$
\operatorname{ker}\left(i_{\zeta}^{*} \omega\right)=\left(T J^{-1}(\zeta)\right)^{\omega} \cap T J^{-1}(\zeta)=\left(S \cap \oplus_{k} \operatorname{Ann}(V)\right)^{\circ} \cap T J^{-1}(\zeta)
$$

Comparing with (4.9), we conclude the following:

Proposition 4.3. The reduced form $\omega_{\text {red }} \in \Omega^{2}\left(M_{\zeta}, \mathbb{R}^{k}\right)$ defined by (4.10) is polysymplectic if and only if

$$
\left(S \cap \oplus_{k} \operatorname{Ann}(V)\right)^{\circ} \cap T J^{-1}(\zeta) \subseteq V_{\zeta}=V \cap T J^{-1}(\zeta) .
$$


A similar, but not equivalent, result of the previous condition was stated on [27, Lemma 3.16].

Example 4.3. Consider symplectic manifolds $\left(M_{j}, \omega_{j}\right)_{j=1, \ldots, k}$ each of them carrying a Hamiltonian action of a Lie group $\mathbb{G}_{j}$ with respective moment map $J_{j}: M_{j} \rightarrow \mathfrak{g}_{j}^{*}$. On the product $k$-poly-symplectic manifold $(M, \omega)$ (see Section 2.1) there is a polysymplectic hamiltonian action given by the product action of $\mathbb{G}:=\prod_{j=1}^{k} \mathbb{G}_{j}$ on $M$ and the moment map $J: M \rightarrow \oplus_{(k)}\left(\prod_{j=1}^{k} \mathfrak{g}_{j}^{*}\right), J(m)=\oplus_{j=1}^{k}\left(0, \ldots, 0, J_{j}\left(m_{j}\right), 0, \ldots, 0\right)$.

Let $\zeta=\oplus_{j=1}^{k}\left(0, \ldots, 0, \zeta_{j}, 0, \ldots, 0\right) \in \oplus_{(k)}\left(\prod_{j=1}^{k} \mathfrak{g}_{j}^{*}\right)$ where $\zeta_{j} \in \mathfrak{g}_{j}^{*}$ is a clean value for $J_{j}$. Then $J^{-1}(\zeta)=\prod_{j=1}^{k} J_{j}^{-1}\left(\zeta_{j}\right)$ and, assuming that each $\mathbb{G}_{\zeta_{j}}$ acts freely and properly on $J_{j}^{-1}\left(\zeta_{j}\right)$, then

$$
M_{\zeta}:=J^{-1}(\zeta) / \mathbb{G}_{\zeta}=\prod_{j=1}^{k} J_{j}^{-1}\left(\zeta_{j}\right) / \mathbb{G}_{\zeta_{j}}=\prod_{j=1}^{k} M_{j, \zeta_{j}},
$$

and the reduced $\mathbb{R}^{k}$-valued 2 -form on $M_{\zeta}$ is the product $k$-poly-symplectic form defined by the reduced symplectic forms on $M_{j, \zeta_{j}}$.

The moment-map reduction of Prop. 4.3 can now be compared with the quotient of poly-Poisson structures in Theorem 4.1 .

Assuming that the $\mathbb{G}$-action on $M$ is free and proper, and that $\zeta$ is a clean value of a moment map $J: M \rightarrow \mathfrak{g}_{(k)}^{*}$, it follows that the $\mathbb{G}_{\zeta^{-}}$action on $J^{-1}(\zeta)$ is also free and proper, and we have the following diagram of submersions and natural inclusions:

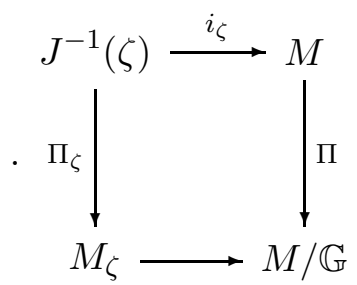

Proposition 4.4. Let $(M, \omega)$ be a poly-symplectic manifold equipped with a hamiltonian $\mathbb{G}$-action with moment map $J: M \rightarrow \mathfrak{g}_{(k)}^{*}$. Assume that the $\mathbb{G}$-action on $M$ is free, proper and reducible (4.2). If $\zeta \in \mathfrak{g}_{(k)}^{*}$ is a clean value for the moment map, then:

(a) The reduced manifold $M_{\zeta}=J^{-1}(\zeta) / \mathbb{G}_{\zeta}$ carries a natural poly-symplectic form defined by equation (4.10);

(b) The poly-symplectic manifold $M_{\zeta}$ sits in $M / \mathbb{G}$ as a union of poly-symplectic leaves of the reduced poly-Poisson manifold on $M / \mathbb{G}$ (given by Thm. 4.1).

Proof. Note that (4.2) (b) directly implies (4.11), so the reduced form $\omega_{\text {red }}$ on $M_{\zeta}$ is indeed poly-symplectic, proving part (a).

By the moment-map condition 4.7(ii), $X \in \operatorname{ker}(d J)$ if and only if $\left(i_{X} \omega\right)\left(u_{M}\right)=0$ for all $u \in \mathfrak{g}$, therefore

$$
\begin{aligned}
T J^{-1}(\zeta) & =\left\{X \in T M \mid i_{X} \omega \in \oplus_{k} \operatorname{Ann}(V)\right\} \\
& =P_{\omega}\left(S_{\omega} \cap \oplus_{k} \operatorname{Ann}(V)\right)=P_{\omega}\left(d \Pi_{(k)}^{*} S_{r e d}\right) .
\end{aligned}
$$


It follows from (4.12) and the construction of the reduced poly-Poisson structure, see (4.4) and (4.5), that

$$
T M_{\zeta}=d \Pi_{\zeta}\left(T J^{-1}(\zeta)\right)=d \Pi\left(P_{\omega}\left(d \Pi_{(k)}^{*} S_{r e d}\right)\right)=P_{r e d}\left(S_{r e d}\right) .
$$

Hence $M_{\zeta}$ is a union of poly-symplectic leaves in $M / \mathbb{G}$. It remains to check that the poly-symplectic structures (the one coming from reduction and the one induced from the poly-Poisson structure on $M / \mathbb{G}$ ) agree.

Consider $\bar{X}=d \Pi_{\zeta}(X), \bar{Y}=d \Pi_{\zeta}(Y) \in T M_{\zeta}$, with $X, Y$ tangent to $J^{-1}(\zeta)$, and let us compute the two 2-forms on them. For the leafwise poly-symplectic form $\omega_{L}$, we have (see (3.6) $)$

$$
\omega_{L}(\bar{X}, \bar{Y})=\bar{\eta}_{r}(\bar{Y})=\left(\Pi_{\zeta}^{*} \omega_{L}\right)(X, Y)
$$

where $\bar{\eta}_{r}$ is such that $\bar{X}=P_{r e d}\left(\bar{\eta}_{r}\right)$. Letting $\bar{\eta}=d \Pi_{(k)}^{*}\left(\bar{\eta}_{r}\right) \in S_{\omega} \cap \oplus_{k} \operatorname{Ann}(V)$, then

$$
\left(\Pi_{\zeta}^{*} \omega_{L}\right)(X, Y)=\bar{\eta}_{r}\left(d \Pi_{\zeta}(Y)\right)=\bar{\eta}(Y)
$$

Note that there exists a unique $X_{0} \in T M$ such that $\bar{\eta}=i_{X_{0}} \omega \in \oplus_{k} \operatorname{Ann}(V)$. By (4.13), we know that $X_{0} \in T J^{-1}(\zeta)$. Furthermore,

$$
d \Pi_{\zeta}\left(X_{0}\right)=d \Pi\left(P_{\omega}(\bar{\eta})\right)=d \Pi\left(P_{\omega}\left(d \Pi_{(k)}^{*}\left(\bar{\eta}_{r}\right)\right)\right)=P_{r e d}\left(\bar{\eta}_{r}\right)=\bar{X},
$$

so $d \Pi_{\zeta}\left(X_{0}\right)=d \Pi_{\zeta}(X)$. Recalling that $\Pi_{\zeta}^{*} \omega_{\text {red }}=i_{\zeta}^{*} \omega$, we see that

$$
\left(\Pi_{\zeta}^{*} \omega_{r e d}\right)(X, Y)=\left(\Pi_{\zeta}^{*} \omega_{r e d}\right)\left(X_{0}, Y\right)=\left(i_{X_{0}} \omega\right)(Y)=\bar{\eta}(Y)=\left(\Pi_{\zeta}^{*} \omega_{L}\right)(X, Y),
$$

showing that $\omega_{\text {red }}=\omega_{L}$ on $M_{\zeta}$.

\section{Example 4.4.}

(a) Let us consider a $\mathbb{G}$-action on $Q$ and its lift to $M=\oplus_{(k)} T^{*} Q$ as in Example 4.1(a). The action on $M$ is hamiltonian, and using the explicit formula for the moment map in Example 4.2 one sees that its poly-symplectic reduction at $\zeta=0$ is $\oplus_{(k)} T^{*}(Q / \mathbb{G})$, with the poly-symplectic form of Example 2.1. Proposition 4.4(b) realizes $\oplus_{(k)} T^{*}(Q / \mathbb{G})$ as a poly-symplectic leaf of $M / \mathbb{G}$.

(b) Following Example 4.1(b), in the particular case $Q=\mathbb{G}$ Proposition 4.4(b) implies that the poly-symplectic reduction of the lifted action on $\oplus_{(k)} T^{*} \mathbb{G}$ at level $\zeta$ (see [27, Sec. 3.3.2]) is identified with the poly-symplectic leaf of $\mathfrak{g}_{(k)}^{*}$ through $\zeta$, which is the orbit of $\zeta$ under the diagonal coadjoint action of $\mathbb{G}$ on $\mathfrak{g}_{(k)}^{*}$ (c.f. Example 3.9) equipped with a poly-symplectic generalization of the usual KKS symplectic form on coadjoint orbits, see [19, Example 2.9] and [27, App. A.3].

4.3. Reduction and integration. In this section, we show (along the lines of [7, 17]) how passing from poly-Poisson manifolds to poly-symplectic groupoids has the effect of turning poly-Poisson actions into hamiltonian actions, and how polysymplectic reduction can be used in the construction of poly-symplectic groupoids associated with poly-Poisson quotients.

In the remainder of this section, we will consider the following set-up:

1. A $k$-poly-Poisson manifold $(M, S, P)$, so that its underlying Lie algebroid is integrable, and $(\mathcal{G} \rightrightarrows M, \omega)$ the source-simply connected $k$-poly-symplectic groupoid integrating it.

2. A poly-Poisson action $\varphi$ of the Lie group $\mathbb{G}$ on $(M, S, P)$. 
Since $\varphi$ preserves the poly-Poisson structure on $M$, the cotangent lift of $\varphi$ induces an action $\hat{\varphi}: \mathbb{G} \times S \rightarrow S$ by Lie-algebroid automorphisms, which can be integrated to a poly-symplectic $\mathbb{G}$-action on $\mathcal{G}$, denoted by

$$
\Phi: \mathbb{G} \times \mathcal{G} \rightarrow \mathcal{G} .
$$

We will now see that this action on $\mathcal{G}$ admits a natural moment map (as in (4.7)), so it is hamiltonian.

Let us start by recalling that any action on $M$ induces a Hamiltonian $\mathbb{G}$-action on the symplectic manifold $T^{*} M$ with moment map $J_{\text {can }}: T^{*} M \rightarrow \mathfrak{g}^{*}$ given by

$$
\left\langle J_{\text {can }}(\alpha), u\right\rangle=\left\langle\alpha, u_{M}\right\rangle
$$

for all $\alpha \in T^{*} M$ and $u$ in the Lie algebra $\mathfrak{g}$ of $\mathbb{G}$. We have an induced map $\oplus_{(k)} T^{*} M \rightarrow \mathfrak{g}_{(k)}^{*}$, that we restrict to $S$ to define

$$
J^{s}: S \rightarrow \mathfrak{g}_{(k)}^{*} \text {. }
$$

It is clear from the $\mathbb{G}$-equivariance of $J_{\text {can }}$ that $J^{s}$ is $\mathbb{G}$-equivariant (with respect to the diagonal coadjoint action on $\left.\mathfrak{g}_{(k)}^{*}\right)$.

The same proof as in [7, Lemma 3.1] shows that, viewing $\mathfrak{g}_{(k)}^{*}$ as a trivial Lie algebra, $J^{s}$ is a Lie-algebroid morphism. According to our sign conventions, it is more convenient to consider $-J^{s}$, which is also a Lie-algebroid morphism, and integrate it to a Lie-groupoid morphism

$$
J: \mathcal{G} \rightarrow \mathfrak{g}_{(k)}^{*} .
$$

Just as in [7, Prop. 3.2] one can verify that $J$ is $\mathbb{G}$-equivariant and satisfies:

$$
i_{u \mathcal{G}} \omega=d\langle J, u\rangle,
$$

for all $u \in \mathfrak{g}$, where $u_{\mathcal{G}}$ is the infinitesimal generator for the action on $\mathcal{G}$. In other words, $J$ is a moment map for the action $\Phi$ on $\mathcal{G}$. The next result summarizes the discussion:

Proposition 4.5. The $\mathbb{G}$-action $\Phi$ on the poly-symplectic groupoid $(\mathcal{G}, \omega)$ is Hamiltonian with moment map (4.15).

We now discuss the connection between integration and reduction. We assume from now on that the $\mathbb{G}$-action $\varphi$ on $M$ is free, proper and reducible (4.2). Then the action $\Phi$ on $\mathcal{G}$ is also free and proper [17, Prop. 4.4]. Let $\left(S_{\text {red }}, P_{\text {red }}\right)$ be the quotient poly-Poisson structure on $M / G$.

Theorem 4.6. Let $0 \in \mathfrak{g}_{(k)}^{*}$ be a clean value for the moment map (4.15). Then $\mathcal{G}_{\text {red }}=$ $J^{-1}(0) / \mathbb{G}$ is a Lie groupoid over $M / \mathbb{G}$, and the reduced form $\omega_{\text {red }} \in \Omega^{2}\left(\mathcal{G}_{\text {red }}, \mathbb{R}^{k}\right)$ makes it into a poly-symplectic groupoid integrating $\left(S_{\text {red }}, P_{\text {red }}\right)$.

Proof. Let $V_{M} \subset T M$ be the vertical bundle with respect to the action on $M$. According with [7, Lemma 3.1] and condition (a) on (4.2) we conclude that $\left(J^{s}\right)^{-1}(0)=$ $S \cap \oplus_{(k)} \operatorname{Ann}\left(V_{M}\right)$ is Lie subalgebroid of $S$. The $\mathbb{G}$-invariance allows us to construct, as in [7, Prop. 4.3], the reduced Lie algebroid $S_{\text {red }}=\left(J^{s}\right)^{-1}(0) / \mathbb{G}$ over $M / \mathbb{G}$. Furthermore, the reduced Lie algebroid $S_{\text {red }}$ coincides the one defined by the reduced poly-Poisson structure of Theorem 4.1

If 0 is a clean value for $J, J^{-1}(0)$ is Lie subgroupoid (see [7, Lemma 5.1]). Following the same lines of [7, Prop. 5.2], we see that $\mathcal{G}_{r e d}=J^{-1}(0) / \mathbb{G}$ is a Lie groupoid over 
$M / \mathbb{G}$, whose Lie algebroid is $\left(S_{r e d}, P_{r e d}\right)$, and the quotient map $\Pi_{0}: J^{-1}(0) \rightarrow \mathcal{G}_{\text {red }}$ is a groupoid morphism.

Let $\omega_{\text {red }}$ be the reduced form on $\mathcal{G}_{\text {red }}$, characterized by $\Pi_{0}^{*} \omega_{\text {red }}=i_{0}^{*} \omega$, where $i_{0}$ is the natural inclusion of $J^{-1}(0)$ on $\mathcal{G}$. The second part of [7, Prop. 5.2] allows us to conclude that $\omega_{\text {red }}$ is multiplicative.

The fact that the quotient map $\Pi_{0}$ and the inclusion $i_{0}$ are groupoid morphism yields

$$
\Pi_{0}^{*}\left(i_{\bar{u}^{R}} \omega_{\text {red }}\right)=i_{u^{R}} \Pi_{0}^{*} \omega_{\text {red }}=i_{0}^{*}\left(i_{u^{R} \omega} \omega\right)
$$

for any $\bar{u} \in S_{\text {red }}$ and $u=d \Pi_{(k)}^{*} \bar{u} \in S \cap \oplus_{(k)} \operatorname{Ann}\left(V_{M}\right)$, where $\bar{u}^{R}$ and $u^{R}$ are the respective right-invariant vector fields on the correspondent Lie groupoid. Moreover, if $t, t_{0}, t_{\text {red }}$ denote the target maps on the Lie groupoids $\mathcal{G}, J^{-1}(0)$ and $\mathcal{G}_{\text {red }}$, respectively, we have

$$
i_{0}^{*}\left(i_{u^{R}} \omega\right)=i_{0}^{*}\left(t^{*} u\right)=t_{0}^{*} d \Pi_{(k)}^{*} \bar{u}=\Pi_{0}^{*} t_{r e d}^{*} \bar{u},
$$

which implies that $i_{\bar{u} R} \omega_{\text {red }}=t_{\text {red }}^{*} \bar{u}$. It follows from Prop. 2.4 that $\omega_{\text {red }}$ is nondegenerate, so $\left(\mathcal{G}_{r e d}, \omega_{\text {red }}\right)$ is a poly-symplectic groupoid, and it integrates $\left(S_{\text {red }}, P_{\text {red }}\right)$.

Theorem 4.6 is a generalization of the following example.

Example 4.5. In Example 3.6 we saw that $\oplus_{(k)} T^{*} Q \rightrightarrows Q$ is the poly-symplectic Lie groupoid integrating the trivial $k$-poly-Poisson structure on $Q$. In this case, for a free and proper $\mathbb{G}$-action on $Q$, the hamiltonian action of Prop. 4.5 is the one induced by cotangent lift, see Example 4.1(a). We conclude that the poly-symplectic reduction in Theorem 4.6 is $\oplus_{(k)} T^{*}(Q / \mathbb{G})$, as in Example 4.4, which is a presymplectic groupoid integrating the trivial poly-Poisson structure on $Q / \mathbb{G}$.

Example 4.6. Recall that for a simply connected manifold $M$, the $k$-poly-symplectic manifold $(M, \omega)$, viewed as poly-Poisson manifold, is integrated by the $s$-simply connected poly-symplectic groupoid $M \times M \rightrightarrows M$ endowed with the poly-symplectic form $t^{*} \omega-s^{*} \omega$, where $t, s$ are the natural projections from $M \times M$ to $M$. If $(M, \omega)$ is equipped with a hamiltonian poly-symplectic action of the Lie group $\mathbb{G}$ and $J_{0}$ : $M \rightarrow \mathfrak{g}_{(k)}^{*}$ is its moment map, then the moment map (4.15) for the hamiltonian action on the groupoid is $J=t^{*} J_{0}-s^{*} J_{0}$. If the action on $M$ is free, proper, reducible and $0 \in \mathfrak{g}_{(k)}^{*}$ is a clean value for $J$, then the symplectic groupoid $J^{-1}(0) / \mathbb{G}$ over $M / \mathbb{G}$ integrates the reduced poly-Poisson structure $\left(S_{\text {red }}, P_{\text {red }}\right)$ induced by $(M, \omega)$.

The poly- symplectic groupoid $\mathcal{G}_{\text {red }}$ in Theorem 4.6 is not necessarily the sourcesimply connected Lie groupoid integrating the reduced structure. This claim is illustrated on [17, Example 4.8] for the case $k=1$.

Remark 4.7. Rather than assuming that 0 is a clean value of the moment map $J$ on $\mathcal{G}$, one can also proceed as in [7, Prop. 5.3] and consider the source-simply-connected groupoid $\mathcal{G}_{0}$ integrating the Lie algebroid $\left(J^{s}\right)^{-1}(0)$. With the same arguments as in [7. Prop. 5.3], one can see that this Lie groupoid is equipped with a $\mathbb{G}$-action and inherits a $\mathbb{G}$-basic multiplicative 2-form $\omega_{0} \in \Omega^{2}\left(\mathcal{G}_{0}, \mathbb{R}^{k}\right)$ from the natural map $\mathcal{G}_{0} \rightarrow$ $\mathcal{G}$, integrating the inclusion $\left(J^{s}\right)^{-1}(0) \rightarrow S$. Then $\mathcal{G}_{0, \text { red }}=\mathcal{G}_{0} / \mathbb{G}$ is a Lie groupoid over $M / \mathbb{G}$ and $\omega_{0}$ reduces to a poly-symplectic form $\omega_{0, \text { red }}$ on $\mathcal{G}_{0, \text { red }}$ integrating the quotient poly-Poisson structure $\left(S_{\text {red }}, P_{\text {red }}\right)$.

Finally, previous remark allows us to conclude that reduced poly-Poisson structure $\left(S_{r e d}, P_{r e d}\right)$ is integrable if the Lie algebroid $(S, P)$ is also integrable. 


\section{REFERENCES}

[1] C. Arias Abad, M. Crainic, The Weil algebra and the Van Est isomorphism, Ann. Inst. Fourier (Grenoble) 61 (2011) 927-970.

[2] A. Awane, k-symplectic structures, J. Math. Phys. 33 (1992)4046-4052.

[3] A. Awane, M. Goze, Pfaffian systems, k-symplectic systems, Kluwer Academic Publishers, Dordrecht (2000).

[4] H. Bursztyn, M. Crainic, A. Weisntein, X. Zhu, Integration of twisted Dirac brackets, Duke Math. J. 123 (2004) 549-607

[5] H. Bursztyn, A. Cabrera, C. Ortiz, Linear and multiplicative 2-forms, Lett. Math. Phys., 90 (2009), 59-83.

[6] H. Bursztyn, A. Cabrera, Multiplicative structure at the infinitesimal level, Math. Ann. 353(2012), 663-705.

[7] H. Bursztyn, A. Cabrera, Symmetries and Reduction of multiplicative 2-forms, J. Geom. Mech., 4 (2012), 111-127.

[8] H. Bursztyn, A. Cabrera, D. Iglesias, Multisymplectic geometry and Lie groupoids, Geometric Mechanics: the Legacy of Jerry Marsden, Fields Institute Communications.

[9] F. Cantrijn, A. Ibort, M. De León, Hamiltonian structures on multisymplectic manifolds, Rend. Sem. Mat. Univ. Pol. Torino, 54 (1996), 225-236. Geom. Struc. for Phys. Theories, I.

[10] F. Cantrijn, A. Ibort, M. De León, On the geometry of multisymplectic manifolds, Journal of the Australian Mathematical Society (Series A), 66, (1999) 303-330.

[11] A. Coste, P. Dazord, A. Weinstein, Groupoïdes symplectiques, Publications du Département de Mathématiques. Nouvelle Série. A, Vol. 2, i-ii, 1-62, Publ. Dép. Math. Nouvelle Sér. A, 87-2, Univ. Claude-Bernard, Lyon, 1987.

[12] T. Courant, Dirac manifolds, Trans. Amer. Math. Soc. 319 (1990), 631-661.

[13] M. Crainic, R. Fernandes, Integrability of Lie brackets, Ann. of Math. 157 (2003), 575-620.

[14] M. Crainic, R. Fernandes, Integrability of Poisson brackets, Journal Differential Geom. 66 Number 1 (2004), 71-137.

[15] M. Crainic, M. Salazar, I. Struchiner, Multiplicative forms and Spencer operators, arXiv:1210.2277

[16] M. Forger, L. Gomes, Multisymplectic and Polysymplectic structures on fiber bundles, Rev. Math. Phys. 25 No.9 (2013) 47 pages.

[17] R.L. Fernandes, J.P. Ortega, T. Ratiu, Momentum maps on Poisson geometry, Amer. J. of Math. 131 (2009), 1261-1310.

[18] C. Gunther, The polysymplectic Hamiltonian formalism in field theory and calculus of variations. I. The local case, J. Differential Geom. Volume 25.

[19] D. Iglesias, J.C Marrero, M. Vaquero, Poly-Poisson Structures, Lett. Math. Phys. 103 (2013) 1103-1133.

[20] I. V. Kanatchikov, Canonical structure of classical field theory in the polymomentum phase space, Rep. Math. Phys. 41(1) (1998) 49-90.

[21] M. de León, I. Méndez, M. Salgado, Regular p-almost cotangent structure, J. Korean Math. Soc 25(2) (1988), 273-287.

[22] M. de León, E. Merino, M. Salgado, k-cosymplectic manifolds and Lagrangian field theories, J. Math. Phys. 42 (2001), 2092.

[23] M. de León S. Vilariño Lagrangian submanifolds in k-symplectic settings, Monatsh Math 170 (2013), 381-404.

[24] D. Li-Bland, AV-Courant algebroids and generalized CR structures, Canad. J. Math. 63 (2011), 938-960.

[25] K.C.H. Mackenzie, General Theory of Lie Groupoids and Lie Algebroids, London Mathematical Society Lecture Note Series, 213 Cambridge : Cambridge University Press, 2005.

[26] K. Mackenzie, P. Xu, Integration of Lie bialgebroids, Topology 39 (2000), 445-467.

[27] J.C Marrero, N. Roman-Roy, M. Salgado, S. Vilariño, Reduction of polysymplectic manifolds, arXiv:1306.0337

[28] G. Martin, A Darboux Theorem for multi-symplectic manifolds, Lett. in Math. Phys. 16 (1988), 133-138. 
[29] M. McLean, L.K. Norris, Covariant field theory on frame bundles of fibered manifolds, J. Math. Phys. 41(10) (2000), 6808.

[30] K. Mikami, A. Weinstein, Moments and reduction for symplectic groupoid actions, Publ. RIMS, Kyoto Univ. 24 (1988), 121-140.

[31] L.K. Norris, Generalized symplectic geometry on the frame bundle of a manifold, Differential geometry: geometry in mathematical physics and related topics (Los Angeles, CA, 1990), pp. 435-465. Proc. Sympos. Pure Math., 54, Part 2 (1993) Amer. Math. Soc., Providence, RI.

[32] L.K. Norris, Symplectic geometry on $T^{*} M$ derived from n-symplectic geometry, L M. J. Geom. Phys. 13(1) (1994) 51-78.

[33] N. Román-Roy, M. Salgado, S Vilariño, Symmetries and conservation laws in the Gunther k-symplectic formailsm of filed Theories Rev. Math. Phys. 19 (2007), 1117.

[34] I. Vaisman, Lectures on the Geometry of Poisson Manifolds, Progress in Mathematics 118: Birkhauser 1994.

[35] A. Weisntein, Symplectic groupoids and Poisson manifolds, Bull. American Mathematical Society 16 (1987), 101-104.

Instituto de Matemática Pura e Aplicada, Estrada Dona Castorina 110, Rio de JANEIRO, 22460-320, BRASIL

E-mail address: nicolasm@impa.br 